\title{
Parking Management Efficiency Analysis through Various Charge Schemes for Day-Long Commuting considering Elastic Travel Demand
}

\author{
Jing Wang $\mathbb{D}^{1},{ }^{1}$ Chaowu Tan $\mathbb{D}^{2,3}$ and Zhigang Liu $\mathbb{D}^{1}$ \\ ${ }^{1}$ College of Urban Railway Transportation, Shanghai University of Engineering Science, 333 Longteng Road, Shanghai 201620, China \\ ${ }^{2}$ College of Transport \& Communications, Shanghai Maritime University, 1550 Haigang Road, Shanghai 201306, China \\ ${ }^{3}$ School of Economics and Management, Tongji University, 1239 Siping Road, Shanghai 200092, China
}

Correspondence should be addressed to Jing Wang; jingwang@sues.edu.cn

Received 15 June 2017; Revised 16 November 2017; Accepted 4 December 2017; Published 23 January 2018

Academic Editor: Ángel Ibeas

Copyright (C) 2018 Jing Wang et al. This is an open access article distributed under the Creative Commons Attribution License, which permits unrestricted use, distribution, and reproduction in any medium, provided the original work is properly cited.

In this paper, we investigate the travel pattern of the day-long commuting in a bisection bottleneck network and the efficiency of pricing schemes with elastic travel demand. We extend the Vickrey model to morning and evening commutes and allow commuters to arrive at workplace late and depart from workplace early. The parking searching time is considered in the morning commute. Next, we derive the independent morning and evening commuting travel patterns without road toll and parking fee. Then, we propose three pricing regimes: duration dependent parking fees; optimal time-varying road tolls; optimal time-varying road tolls with duration dependent parking fees. We compare the efficiency of the four schemes with elastic demand. Theoretical analysis and the numerical examples show that optimal time-varying road toll is the most efficient pricing scheme. Charging a duration dependent fee neither improves nor deteriorates the scheme of time-varying road toll, if the toll rates are appropriately set. The regime of duration dependent parking fee only is less efficient than the regime of independent morning and evening commuting travel patterns without road toll and parking fee. In the regime of duration dependent parking fee, the social surplus decreases with the increase of duration dependent parking fee rate.

\section{Introduction}

With the development of metropolis and process of urbanization, traffic demand is increasing steadily. Expanding road network is not efficient, due to the limitation of budget and urban land. Sometimes network expansion is very complicated due to the involvement of various sectors [1]. Parking management provides a new method for urban traffic management. In morning commuting, parking supply cannot meet the traffic demand especially in downtown area and people have to spend much cost for acquiring parking spot. Many scholars have investigated the parking management in morning commuting. Arnott et al. [2] proposed a model that considers spatial distribution of parking in morning commuting and compared the efficiency of road toll and parking fees regimes. Bifulco [3] presented a stochastic user equilibrium assignment model to evaluate parking policies
Verhoef et al. [4] studied the parking policies as a substitute to road pricing. Zhang et al. [5] used parking permits to improve the traffic efficiency by eliminating the competition for inadequate parking spots. Qian et al. [6] studied the economics of parking provision for the morning commuting. However, there are few researches in integral analysis of parking searching time for parking space and bottleneck dynamic. In this paper, we analyze how the parking searching time and parking fees affect the commuter's behavior and examine the efficiency of road toll and parking fee to improve the social surplus with elastic demand.

The basic model used in our paper is originated by Vickrey [7]; in the morning, commuters need go to workplace in central business district through a bottleneck with limited service capacity. In the equilibrium, no one can reduce individual travel cost by changing the departure time. Later, many scholars made a lot of research based the bottleneck model. 
Daganzo [8] assumed commuters have different working start time and proved uniqueness of the time-dependent equilibrium distribution of arrivals at a single bottleneck. Arnott et al. [9] extended the bottleneck model from fixed demand to elastic demand. Zhang et al. [10] investigated how travelers behave and react on bottlenecks with time-varying capacities, examined the user equilibrium and system optimal traffic patterns, and derived pricing regimes that lead to the system optimum pattern. Recently, many scholars started to measure traffic flows of downtown area. Geroliminis and Daganzo [11] demonstrated the existence of the macroscopic fundamental diagram (MFD), correlating the rate of ending trips with the number of vehicles in an urban area. Arnott [12] assumed that the outflow and the travel time from the downtown area depend on the vehicle accumulation at bottleneck and developed a bathtub model of downtown traffic congestion on the basis of MFD. Fosgerau [13] proposed a similar bathtub model considering the heterogeneity in trip length of population. Geroliminis [14] integrated the traffic dynamic of cruising for parking with a spatially aggregated model of urban hypercongestion, the macroscopic fundamental diagram. Following the study of Geroliminis [14], Liu and Geroliminis [15] explored how the interactions between cruising for parking and congestion reshape the morning commuting considering travelers' scheduling cost and time of departure choice. Ji et al. [16] considered the parking behavior of mixed autonomous and traditional vehicles in a bottleneck and investigated the effect of fare rate of autonomous vehicles. Jiang [17] found that automatic driving can substantially reduce the queuing delay at the intersection bottlenecks. In this paper, we use the traditional queuing model and Vickrey model and treat capacity of bottleneck as a constant value.

The past researches mainly focused on the morning commuting; the evening commuting was not often considered. It was generally believed that the traffic pattern in evening commuting is a mirror image of that in morning commuting. de Palma and Lindsey [18] compared the morning and evening commutes and differed them in just one respect that the schedule preference in morning commuting is in terms of arrival time in destination and the preference in evening is in terms of departure time from destination. Daganzo [19] established the system optimum of day-long commuting and minimized the general social cost of whole day with two travel modes: car and transit. He assumed that the commuters have different wished arrival time and departure time. Gonzales and Daganzo [20] showed that the user equilibrium for isolated morning and evening commutes are asymmetric for different schedule penalty in morning and evening commutes. Zhang et al. [5], Yang et al. [21], and Wang et al. [22] developed methods of parking permit distribution and trading to improve travel efficiency and reduce traffic emission.

Motivated by Arnott et al. [2], Zhang et al. [23] derived a model integrating morning and evening commutes and developed a time-varying road toll and location-dependent parking fee regime to achieve system optimum. Zhang and van Wee [24] compared efficiency of several parking schemes and solved the optimal parking fee rate by minimizing the

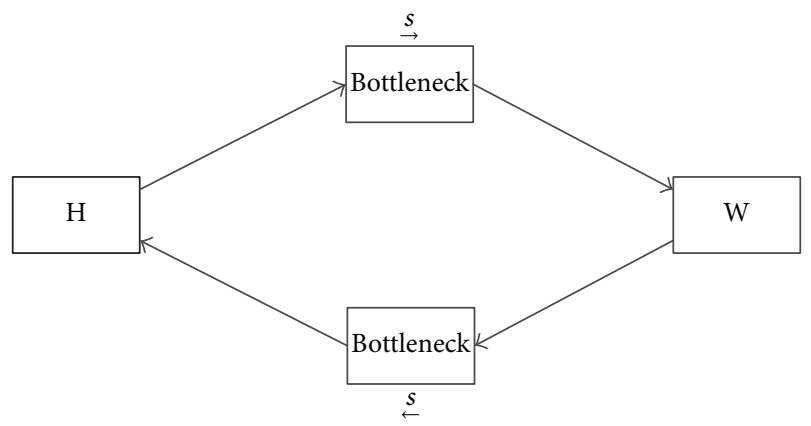

FIGURE 1: A bidirection bottleneck network in morning and evening commutes.

social cost or maximizing social surplus when considering parking searching time.

In this paper, Vickrey's model is developed from a single morning peak to morning and evening peaks. The morning and evening commutes are treated as two independent user equilibrium traffic patterns for the day-long commuting. The analysis of this paper is based on a single bottleneck; it is expected that we can consider day-long parking behavior with network effect with resorting to some approximation methods [25]. Besides, we relax the assumption in the study of Zhang and van Wee [24] by allowing the commuters to arrive at workplace late in morning commuting and leave workplace early in evening commuting. Moreover, traffic demands are often stochastic [26] and dynamic [25]; therefore it is very difficult to precisely predict the travel demands. Travel demands are often influenced by some social and economic factors and affected by travel costs. In the paper, we extend the analysis to considering elastic demands.

Figure 1 shows the network of day-long commuting. In Figure 1, home is represented by " $\mathrm{H}$ " and workplace is represented by "W." Let $N$ denote the number of commuters who travel from home to workplace in the morning and return from workplace to home in the evening. The service capacity of bottleneck in home-to-work direction is $\stackrel{s}{\rightarrow}$ and that in work-to-home direction is $s$. We assume the parking lot is near to workplace and ignore the commuters' walking time between parking spots and workplace. However, searching time of parking spots is considered in morning commuting. We assume that there is no information provided for auto drivers in the parking lot and the parking searching time increases as the number of occupied parking spots increases with a fixed rate $\pi$. Here, $\pi$ is the increasing searching time rate of unit parking spot. Then the searching time for parking spots in morning commuting with $n$ occupied parking spots is $\pi n$. So the first commuter has no parking searching time and the last commuter's parking searching time is $\pi N$. When commuters leave from workplace in evening commuting, their searching time for parking spots is zero because they have known where their cars are parked in the morning.

In morning and evening commutes, the commuters have an identical desired arrival time to workplace $\underset{t^{*}}{\rightarrow}$ and a departing time from workplace $t^{*}$. In the morning, if 
commuters cannot arrive at workplace on time, there are schedule delay costs for them. Let $\beta_{1}$ be the cost of unit early arrival time and $\gamma_{1}$ be the cost of unit late arrival time. In the evening, the commuters also have schedule delay costs for early departing or late departing from workplace. The cost of unit early departing time is $\beta_{2}$ and the cost of unit late departing time is $\gamma_{2}$. The cost of unit travel time is $\alpha$ for both morning and evening commutes. Following previous related studies [18, 27], it is assumed that $\gamma_{1}>\alpha>\beta_{1}$ and $\beta_{2}>\alpha>$ $\gamma_{2}$. For simplifying the model in this paper, we assume the morning commuting and evening commuting are symmetric. It means that the cost of unit early arrival time in morning commuting $\beta_{1}$ is equivalent to the cost of unit late departing time in evening commuting $\gamma_{2}$; the cost of unit late arrival time in morning commuting $\gamma_{1}$ is equivalent to the unit early departing time in evening commuting $\beta_{2}$ and the capacity of bottleneck in home-to-work direction is equivalent to that in work-to-home direction, $s=s$.

When there is no toll and parking fee, the individual travel time includes queuing time and searching time for parking spots in morning commuting. However, in evening commuting, the travel time only comprises queuing time. As commuter has known the parking spot, searching time is zero in evening commuting. Besides, we ignore the walking time from parking lot to working place. The free flow travel time spent in home to bottleneck is assumed to be zero in morning, and that in the reverse direction is also zero. It means that commuter arrives to the bottleneck as soon as he leaves home in morning commuting and commuter arrives to bottleneck as soon as he leaves from workplace in evening commuting.

In this paper, we investigate the traffic patterns in morning and evening commutes under various regimes and compare their respective efficiency with elastic demand. The four regimes proposed in this paper are listed as follows:

Regime $f$ : user equilibrium without road toll and parking fee;

Regime $u$ : duration dependent parking fees;

Regime $r$ : optimal time-varying road tolls;

Regime $o$ : optimal time-varying road tolls with duration dependent parking fees.

We present the notations used in this paper to describe traffic pattern of four regimes in Notations.

\section{User Equilibrium without Road Toll and Parking Fee (Regime $f$ )}

2.1. User Equilibrium in Evening Commuting. In evening commuting, commuters do not need to search parking spots; the individual travel cost $C_{e}^{f}$ is a function of the departing time from workplace $t^{f}$. In user equilibrium, the individual travel cost cannot further decrease by changing departing time from workplace. So, we first give the individual travel cost of a commuter departing workplace early:

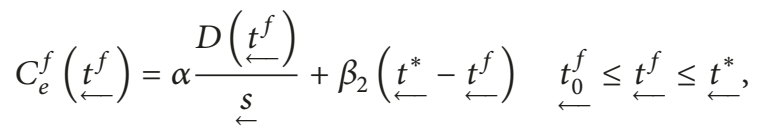

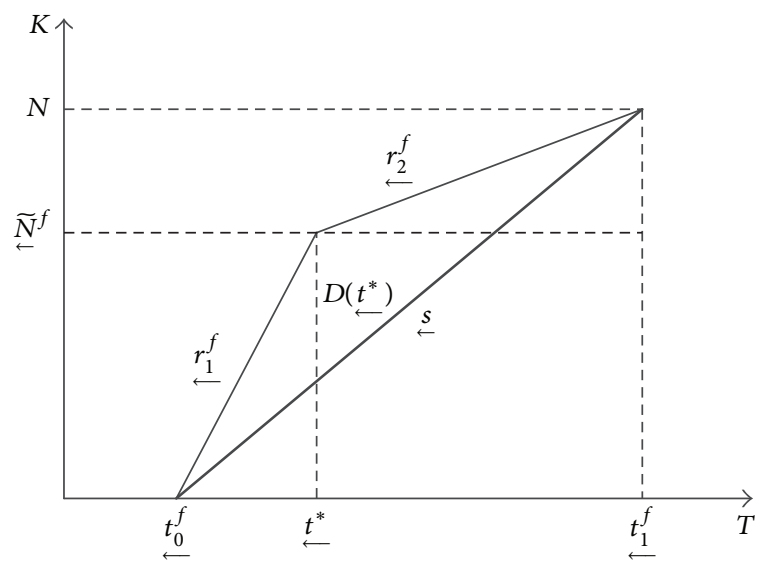

FIGURE 2: User equilibrium in evening commuting of regime $f$.

where $t_{0}^{f}$ is the time at which the first commuter leaves from workplace and $D\left(t^{f}\right)$ is the queue length. The first term of right hand of (1) is the cost of waiting time at bottleneck and the second term is the schedule delay cost.

For a commuter departing workplace late, the individual travel cost is

$$
C_{e}^{f}\left(\stackrel{t^{f}}{\longleftarrow}\right)=\alpha \frac{D\left(\stackrel{t^{f}}{\longleftarrow}\right)}{s}+\gamma_{2}\left(\stackrel{t}{t}^{f}-t^{t^{*}}\right) \quad t^{*} \leq t^{f} \leq \longleftarrow t_{1}^{f} .
$$

Here $t_{1}^{f}$ is the departing time from workplace of the last commuter. According to user equilibrium condition in evening commuting, $d C_{e}^{f}(\underbrace{t^{f}}) / d t^{f}=0$, we can get

$$
\frac{d D\left(\frac{t^{f}}{\longleftarrow}\right)}{d t^{f}}= \begin{cases}\stackrel{s}{\longleftarrow} \beta_{2} & t_{0}^{f} \leq t^{f} \leq t^{*} \\ \longleftarrow & \longleftarrow \\ -\frac{s}{\alpha} \gamma_{2} & \stackrel{t^{*}}{\longleftarrow} \leq t^{f} \leq t_{1}^{f} .\end{cases}
$$

According to (3), we can easily obtain the departure rate from workplace $r_{1}^{f}, r_{2}^{f}, r_{1}^{f}=\left(\beta_{2} / \alpha+1\right) \underset{\leftarrow}{s}, r_{2}^{f}=(1-$ $\left.\gamma_{2} / \alpha\right) s$. Obviously, in the evening commuting in regime $f$, the departure rate from workplace of early departing $r_{1}^{f}$ is larger than capacity of bottleneck in work-to-home direction, $r_{1}^{f}>s$, and the departure rate from workplace of late departing $r_{2}^{f}$ is less than $\underset{\leftarrow}{\leftarrow} r_{1}^{f}<\underset{\leftarrow}{\leftarrow}$. Then the capacity of bottleneck is fully used in the evening commuting and the beginning time and ending time of evening peak satisfy the equation as follows:

$$
\stackrel{t_{1}^{f}}{\longleftarrow}-\underset{t_{0}^{f}}{\longleftarrow}=\frac{N}{s}
$$

The user equilibrium depicted in Figure 2 requires that the individual travel cost of the first commuter is equivalent to 
that of the last commuter, $C_{e}^{f}\left(t_{0}^{f}\right)=C_{e}^{f}\left(t_{1}^{f}\right)$, so the departure times of the first and last commuter are

$$
\begin{aligned}
& t_{0}^{f}=t^{*}-\left(\frac{\gamma_{2}}{\beta_{2}+\gamma_{2}}\right) \underset{\leftarrow}{\stackrel{N}{s}}, \\
& \stackrel{t_{1}^{f}}{\longleftarrow} \underbrace{t^{*}}+\left(\frac{\beta_{2}}{\beta_{2}+\gamma_{2}}\right) \underset{\stackrel{N}{s}}{\leftarrow} .
\end{aligned}
$$

The individual travel cost in evening commuting of regime $f$ is

$$
C_{e}^{f}=\frac{\beta_{2} \gamma_{2}}{\beta_{2}+\gamma_{2}} \stackrel{N}{\underset{s}{\leftarrow}}
$$

The system cost in evening commuting of regime $f$ is

$$
\mathrm{SC}_{e}^{f}=\frac{\beta_{2} \gamma_{2}}{\beta_{2}+\gamma_{2}} \frac{N^{2}}{\stackrel{s}{\leftarrow}} .
$$

In Figure 2, the departing rate from workplace of early departing commuters is $r_{1}^{f}$ and the departing rate of late departing commuter is $r_{2}^{f}$. The capacity of bottleneck is $\stackrel{s}{\longleftarrow}$ The number of early departing commuters is $\tilde{N}^{f}$. For the commuter who departs from workplace on time, the queue length at bottleneck is $D\left(t^{*}\right)$ and the waiting time is $D\left(t^{*}\right) / s$.

2.2. User Equilibrium in Morning Commuting. In morning commuting, the travel time includes queuing time and the searching time for parking spot. The individual travel cost is a function with respect to the leaving time from bottleneck $\underset{t^{f}}{\longrightarrow}$. The individual travel cost for the commuter arriving to workplace early is

$$
\begin{aligned}
& C_{m}^{f}\left(\stackrel{t^{f}}{\rightarrow}\right)=\alpha\left[\frac{D\left(\stackrel{t^{f}}{\rightarrow}\right)}{\stackrel{s}{\rightarrow}}+\pi \underset{s}{\rightarrow}\left(\stackrel{t^{f}}{\rightarrow}-\stackrel{t_{0}^{f}}{\rightarrow}\right)\right] \\
& +\beta_{1}\left[\stackrel{t^{*}}{\longrightarrow}-\stackrel{t^{f}}{\longrightarrow}-\pi \stackrel{s}{\rightarrow}\left(\stackrel{t^{f}}{\longrightarrow}-\stackrel{t_{0}^{f}}{\rightarrow}\right)\right] .
\end{aligned}
$$

Here, the arrival time of the first commuter to parking lot is $t_{0}^{f}$. The queuing time at bottleneck is $D\left(\stackrel{t^{f}}{\rightarrow}\right) / \stackrel{s}{\rightarrow}$ and the searching time for parking spot is $\pi \underset{s}{\rightarrow} \stackrel{t^{f}}{\longrightarrow}-\underset{t_{0}^{f}}{\longrightarrow}$.

The individual travel cost for the commuter who arrives to the workplace late is

$$
\begin{aligned}
C_{m}^{f}= & \alpha\left[\frac{D\left(\stackrel{t^{f}}{\rightarrow}\right)}{\stackrel{s}{\rightarrow}}+\pi s\left(\stackrel{t^{f}}{\rightarrow}-\stackrel{t_{0}^{f}}{\rightarrow}\right)\right] \\
& +\gamma_{1}\left[\stackrel{t^{f}}{\rightarrow}+\pi \stackrel{s}{\rightarrow}\left(\stackrel{t^{f}}{\longrightarrow}-\underline{t_{0}^{f}}\right)-\underline{t^{*}} \rightarrow .\right.
\end{aligned}
$$

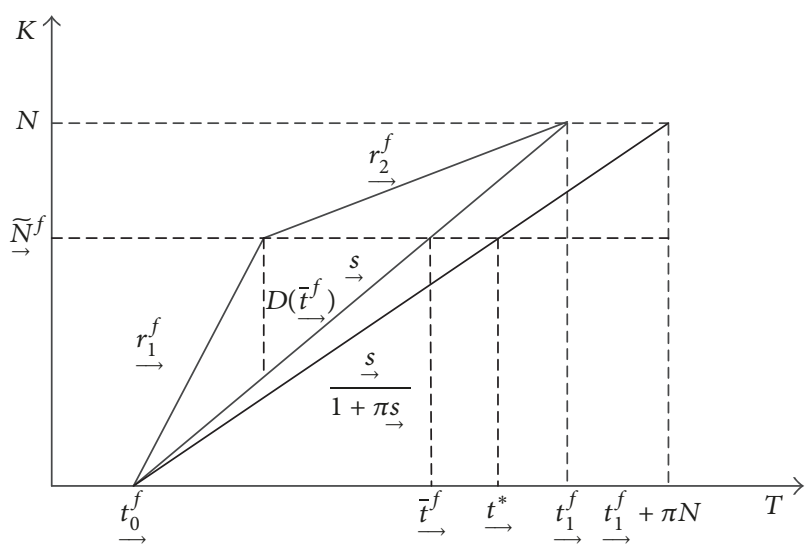

FIGURE 3: User equilibrium in morning commuting of regime $f$.

According to the equilibrium condition of morning commuting $d C_{m}^{f}\left(\stackrel{t^{f}}{\rightarrow}\right) / d \underline{t^{f}}=0$, we can get

$$
\begin{aligned}
& \frac{d D\left(\underline{t^{f}}\right)}{d \stackrel{f t}{\longrightarrow}}
\end{aligned}
$$

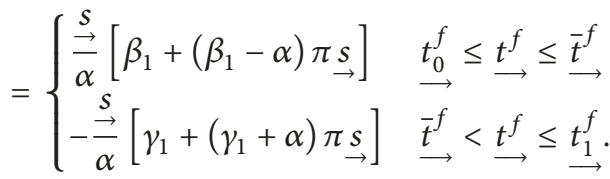

Here, we hold the same assumption $\beta_{1}(1+\pi s)>\alpha \pi s$ s the study of Arnott et al. [2] to ensure a growing queue at the bottleneck. $\bar{t}^{f}$ is the leaving time from bottleneck of the commuter who arrives at workplace on time. The arrival rate to bottleneck $r_{1}^{f}$ and $r_{2}^{f}$ can be obtained easily, $r_{1}^{f}=\alpha \underset{\rightarrow}{s} /(\alpha-$ $\left.\left[\beta_{1}+\left(\beta_{1}-\alpha\right) \pi s\right]\right), \underset{\rightarrow}{r_{2}^{f}}=\alpha \underset{s}{s} /\left(\alpha+\left[\gamma_{1}+\left(\gamma_{1}+\alpha\right) \pi \underset{s}{s}\right]\right)$.

Figure 3 shows user equilibrium in the morning commuting of regime $f$. The length of rush hour interval is $N / s$, $t_{1}^{f}-t_{0}^{f}=N / \stackrel{s}{\rightarrow}$. The equilibrium condition requires the travel cost of the first commuter to be equivalent to that of the last commuter:

$$
\beta_{1}\left(\stackrel{t^{*}}{\longrightarrow}-\stackrel{t_{0}^{f}}{\longrightarrow}\right)=\gamma_{1}\left(\stackrel{t_{1}^{f}}{\longrightarrow}+\pi N-\underline{t^{*}}\right)+\alpha \pi N
$$

So we can easily get the leaving times from bottleneck of the first commuter and last commuter:

$$
\begin{aligned}
& \stackrel{t_{0}^{f}}{\rightarrow}=t^{*}-\frac{\gamma_{1}+\left(\alpha+\gamma_{1}\right) \pi \stackrel{s}{\rightarrow}}{\beta_{1}+\gamma_{1}} \frac{N}{\stackrel{s}{\rightarrow}}, \\
& \stackrel{t_{1}^{f}}{\rightarrow} t^{*}+\frac{\beta_{1}-\left(\alpha+\gamma_{1}\right) \pi \stackrel{s}{\rightarrow}}{\beta_{1}+\gamma_{1}} \frac{N}{\rightarrow}
\end{aligned} .
$$


The individual travel cost in morning commuting of regime $f$ is

$$
C_{m}^{f}=\frac{\beta_{1} \gamma_{1}+\beta_{1}\left(\alpha+\gamma_{1}\right) \pi \stackrel{s}{\rightarrow}}{\beta_{1}+\gamma_{1}} \frac{N}{\stackrel{s}{\rightarrow}} .
$$

The system cost in morning commuting of regime $f$ is

$$
\mathrm{SC}_{m}^{f}=\frac{\beta_{1} \gamma_{1}+\beta_{1} \pi \underset{\rightarrow}{\rightarrow}\left(\alpha+\gamma_{1}\right)}{\beta_{1}+\gamma_{1}} \frac{N^{2}}{\stackrel{s}{\rightarrow}} .
$$

In Figure 3, the arrival rate to bottleneck of early arrival commuters is $\underset{1}{r_{1}^{f}}$ and the arrival rate to bottleneck of late arrival commuters is $r_{2}^{f}$. The number of early arrival commuters is $\stackrel{\tilde{N}}{\rightarrow}^{f}$. The capacity of bottleneck is $\stackrel{s}{\rightarrow}$ and the arrival rate to the workplace is $\underset{\rightarrow}{s} /(1+\pi s)$. For the commuter who arrives at workplace on time, the queue length is $D\left(\bar{t}^{f}\right)$ and the searching time for parking is $\pi \underset{s}{\rightarrow}\left(\bar{t}^{f}-\underset{t_{0}^{f}}{\rightarrow}\right.$. In the user equilibrium, the commuter cannot reduce individual travel cost by changing departing time.

\section{Duration Dependent Parking Fees (Regime $u$ )}

A continuous duration parking fee usually equals the parking duration time multiplied by charge rate $\mu$ [28]. We assume that parking fees are charged based on the parking duration given a uniform parking fee rate $\mu$. Parking costs are assigned to the morning and evening trips, respectively, using an intermediate time point $t^{\Delta}, t^{\Delta}=\left(t^{*}-t^{*}\right) / 2$, [5]; $t^{\Delta}$ is denoted as the midday time in this section.

Using the analytic method shown in Appendix A, we can obtain the departure rate from workplace in evening commuting $r_{1}^{u}(\mu), r_{2}^{u}(\mu), r_{1}^{u}(\mu)=\underset{\leftarrow}{s}\left(1+\left(\beta_{2}-\mu\right) / \alpha\right), r_{2}^{u}(\mu)=$ $s\left(1-\left(\gamma_{2}+\mu\right) / \alpha\right)$. Here, we require the parking fee rate $\mu$ not to exceed $\beta_{2}, \mu<\beta_{2}$, to ensure the growing queue at bottleneck. Then, in regime $u$, we have $r_{1}^{u}(\mu)>\underset{s}{s}, r_{2}^{u}(\mu)<s$.

The first commuter's departure time and the last commuter's departure time are

$$
\begin{aligned}
& t_{0}^{u}(\mu)=t^{*}-\left(\frac{\gamma_{2}+\mu}{\beta_{2}+\gamma_{2}}\right) \frac{N}{s}, \\
& t_{1}^{u}(\mu)=t^{t^{*}}+\left(\frac{\beta_{2}-\mu}{\beta_{2}+\gamma_{2}}\right) \frac{N}{\stackrel{N}{\leftarrow}} .
\end{aligned}
$$

In evening commuting, because commuters in regime $u$ may depart earlier than regime $f$ to reduce their parking fees, the leaving time from workplace is advanced as the commuter's parking fee rate $\mu$ increases. From (15), we can find that, in the user equilibrium of regime $u$, the starting time and ending time of leaving from workplace will be advanced $\left(\mu /\left(\beta_{2}+\right.\right.$
$\left.\left.\gamma_{2}\right)\right)(N / \stackrel{s}{\leftarrow})$ on the basis of that in regime $f, \underbrace{u}_{0}(\mu)-\underbrace{f}_{0}=$ $\left(\mu /\left(\beta_{2}+\gamma_{2}\right)\right)(N / s)$.

The individual travel cost in evening commuting of regime $u$ is

$$
C_{e}^{u}(\mu)=\frac{\beta_{2} \gamma_{2}+\left(\beta_{2}-\gamma_{2}\right) \mu-\mu^{2}}{\beta_{2}+\gamma_{2}} \frac{N}{s}+\mu\left(t^{t^{*}}-t^{\Delta}\right) .
$$

The system travel cost in evening commuting of regime $u$ is

$$
\begin{aligned}
& \mathrm{SC}_{e}^{u}(\mu)=\frac{1}{2} \\
& \cdot \frac{\left[2 \alpha \beta_{2} \gamma_{2}+\beta_{2} \gamma_{2} \mu+\left(\beta_{2}-\gamma_{2}\right)\left(\mu^{2}+\alpha \mu\right)-\mu^{3}\right]}{\alpha\left(\beta_{2}+\gamma_{2}\right)} \\
& \cdot \frac{N^{2}}{s} .
\end{aligned}
$$

Similarly, the arrival rate of early and late arrival to bottleneck in morning commuting, $r_{1}^{u}(\mu)$ and $r_{2}^{u}(\mu)$ are $r_{1}^{u}(\mu)=$ $\alpha \underset{\rightarrow}{s} /\left(\alpha-\beta_{1}-\mu\right)(1+\pi \underset{\rightarrow}{s}), r_{2}^{u}(\mu)=\underset{\rightarrow}{\vec{s}} /\left(\alpha+\gamma_{1}-\mu\right)(1+$ $\pi \stackrel{s}{\rightarrow}$. Obviously, the departure rates from home $\underset{r_{1}^{u}}{\longrightarrow}(\mu), \stackrel{r_{2}^{u}}{\longrightarrow}(\mu)$ increase as the parking fee rate $\mu$ increases, and the departure rate in regime $u$ is not less than that in regime $f, r_{1}^{u}(\mu) \geq$ $\stackrel{r_{1}^{f}}{\rightarrow} \underset{2}{r_{2}^{u}}(\mu) \geq \underline{r_{2}^{f}}$.

In regime $u$, if $\mu>\alpha-\beta_{1}$, the departure rate $r_{1}^{u}(\mu)$ is negative and if $\mu=\alpha-\beta_{1}$, the departure rate $r_{1}^{u}(\mu)$ is infinity. So we can set $0 \leq \mu<a-\beta_{1}$ to ensure the existence of equilibrium of morning commuting. Then we have $\underset{r_{1}^{u}}{\longrightarrow}(\mu)>$ $\stackrel{s,}{\rightarrow} \underset{r_{2}^{u}}{\rightarrow}(\mu)<\stackrel{s}{\rightarrow}$.

The arrival times to parking lot of the first and last commuter are

$$
\begin{aligned}
& \stackrel{t_{0}^{u}}{\rightarrow}(\mu)=\stackrel{t^{*}}{\rightarrow}+\frac{\mu-\gamma_{1}}{\beta_{1}+\gamma_{1}} \frac{N}{\stackrel{s}{\rightarrow}}+\frac{\mu-\gamma_{1}}{\beta_{1}+\gamma_{1}} \pi N-\frac{\alpha \pi N}{\beta_{1}+\gamma_{1}}, \\
& \stackrel{t_{1}^{u}}{\longrightarrow}(\mu)=\stackrel{t^{*}}{\rightarrow}+\frac{\beta_{1}+\mu}{\beta_{1}+\gamma_{1}} \frac{N}{\stackrel{s}{\rightarrow}}+\frac{\mu-\gamma_{1}}{\beta_{1}+\gamma_{1}} \pi N-\frac{\alpha \pi N}{\beta_{1}+\gamma_{1}} .
\end{aligned}
$$

We can find that, in morning commuting of regime $u$, commuters can delay their arrival time to workplace to reduce the parking fee. So, commuters will put the arrival time backward till no commuter can reduce individual travel cost. The interval of morning rush hour in regime $u$ will be delayed by $\left(\mu(1+\pi s) /\left(\beta_{1}+\gamma_{1}\right)\right)(N / s)$ comparing with that in regime $f, \stackrel{t_{0}^{u}}{\rightarrow}(\mu)-\underline{t_{0}^{f}}=\left(\mu(1+\pi s) /\left(\beta_{1}+\gamma_{1}\right)\right)(N / \stackrel{s}{\rightarrow})$. 
The individual travel cost in morning commuting of regime $u$ is

$$
\begin{aligned}
C_{m}^{u}(\mu)= & \frac{\left(\beta_{1}+\mu\right)\left(\gamma_{1}-\mu\right)}{\beta_{1}+\gamma_{1}} \frac{N}{\stackrel{N}{\rightarrow}}(1+\pi \underset{s}{s}) \\
& +\frac{\alpha \pi N}{\beta_{1}+\gamma_{1}}\left(\beta_{1}+\mu\right)+\mu\left(t^{\Delta}-\underset{t^{*}}{\rightarrow}\right) .
\end{aligned}
$$

The system cost in morning commuting of regime $u$ is

$$
\begin{aligned}
\operatorname{SC}_{m}^{u}(\mu)= & \frac{\beta_{1}\left(\gamma_{1}-\mu\right)}{\beta_{1}+\gamma_{1}} \frac{N^{2}}{\stackrel{s}{\rightarrow}}(1+\pi \underset{\rightarrow}{s})+\frac{\alpha \beta_{1} \pi N^{2}}{\beta_{1}+\gamma_{1}} \\
& +\frac{1}{2} \mu \frac{N^{2}}{\stackrel{s}{\rightarrow}}(1+\pi \underset{\rightarrow}{s}) .
\end{aligned}
$$

According to (16) and (19), we can find that the midday time $t^{\Delta}$ cannot affect the total individual travel cost of daylong commuting and the total system cost. However, the midday time can determine the ratio of morning commuting individual travel cost $C_{m}^{u}(\mu)$ (or evening commuting) to the total individual travel cost $C_{m}^{u}(\mu)+C_{e}^{u}(\mu)$.

\section{Optimal Time-Varying Road Tolls (Regime $r$ )}

In regime $r$, the queue delay at bottleneck can be eliminated by time-varying road toll. Then, the system cost is equivalent to the total schedule delay cost and the optimal time rush hour can be obtained by adjusting road toll to minimize the total schedule delay cost.

Using a similar analytic method shown in Appendix B, we can get the toll function $\tau\left(t^{r}\right)$ with respect to the departing time from workplace $t^{r}$ in the evening commuting:

$$
\begin{aligned}
& \tau\left(\begin{array}{l}
t^{r} \\
\longleftarrow
\end{array}\right) \\
& \quad= \begin{cases}\frac{\beta_{2} \gamma_{2}}{\beta_{2}+\gamma_{2}} \frac{N}{\leftarrow}+\beta_{2}\left(t^{t^{r}}-t^{*}\right) & t_{0}^{r} \leq t^{r} \leq t^{*}, \\
\frac{\beta_{2} \gamma_{2}}{\beta_{2}+\gamma_{2}} \frac{N}{\leftarrow}-\gamma_{2}\left(t^{r}-t^{*}\right) & \stackrel{t^{*}}{\longleftarrow}<t^{r} \leq \stackrel{t_{1}^{r} .}{\longleftarrow}\end{cases}
\end{aligned}
$$

In the equilibrium shown in Figure 4, the departing times of first and last commuters are

$$
\begin{aligned}
& t_{0}^{r}=t^{*}-\frac{\gamma_{2}}{\beta_{2}+\gamma_{2}} \frac{N}{\stackrel{s}{\leftarrow}}, \\
& t_{1}^{r}=t^{*}+\frac{\beta_{2}}{\beta_{2}+\gamma_{2}} \frac{N}{\leftarrow} .
\end{aligned}
$$

In Figure 4, with the existence of time-varying road toll, the queue is eliminated and the departure rates of early and late departing commuter are equivalent to the capacity $s$.

The individual travel cost in evening commuting of regime $r$ is

$$
C_{e}^{r}=\frac{\beta_{2} \gamma_{2}}{\beta_{2}+\gamma_{2}} \frac{N}{\stackrel{N}{\leftarrow}}
$$

The system cost in evening commuting of regime $r$ is

$$
\mathrm{SC}_{e}^{r}=\frac{1}{2} \frac{\beta_{2} \gamma_{2}}{\beta_{2}+\gamma_{2}} \frac{N^{2}}{\stackrel{s}{\leftarrow}} \text {. }
$$

Using a similar method, we can also obtain the optimal toll function in morning commuting which is given as follows:

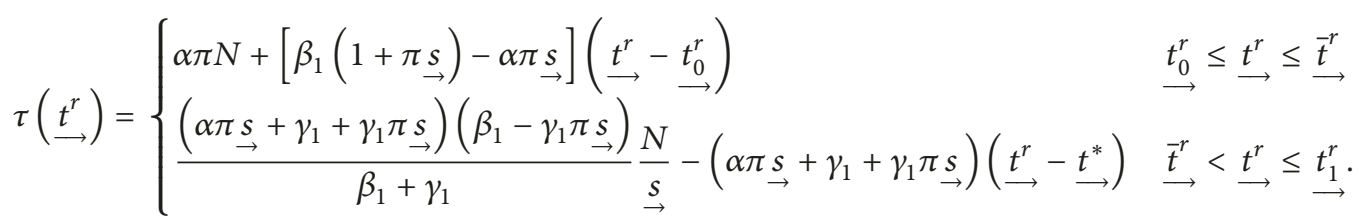

In the user equilibrium shown in Figure 5, the arrival times to parking lot of the first and last commuter are

$$
\begin{aligned}
& \stackrel{t_{0}^{r}}{\rightarrow} \stackrel{t^{*}}{\rightarrow}-\frac{\gamma_{1}(1+\pi \underset{s}{\rightarrow})}{\beta_{1}+\gamma_{1}} \frac{N}{s}, \\
& \stackrel{t_{1}^{r}}{\rightarrow} \stackrel{t^{*}}{\rightarrow}+\frac{\beta_{1}-\gamma_{1} \pi \stackrel{s}{\rightarrow}}{\beta_{1}+\gamma_{1}} \underset{\stackrel{s}{s}}{\rightarrow}
\end{aligned}
$$

In Figure 5, the queue at bottleneck in morning commuting is also eliminated by time-varying road toll. With the existence of parking searching time, the arrival rate to workplace is $\stackrel{s}{\rightarrow} /(1+\pi \underset{s}{\rightarrow})$.

The individual travel cost in morning commuting of regime $r$ is

$$
C_{m}^{r}=\frac{\beta_{1} \gamma_{1}(1+\pi \underset{s}{\rightarrow})}{\beta_{1}+\gamma_{1}} \frac{N}{\stackrel{s}{\rightarrow}}+\alpha \pi N .
$$

The system cost in morning commuting of regime $r$ is

$$
\mathrm{SC}_{m}^{r}=\frac{1}{2} \alpha \pi N^{2}+\frac{1}{2} \frac{\beta_{1} \gamma_{1}}{\beta_{1}+\gamma_{1}} \frac{N^{2}}{\stackrel{s}{\rightarrow}}(1+\pi \underset{\rightarrow}{s}) \text {. }
$$




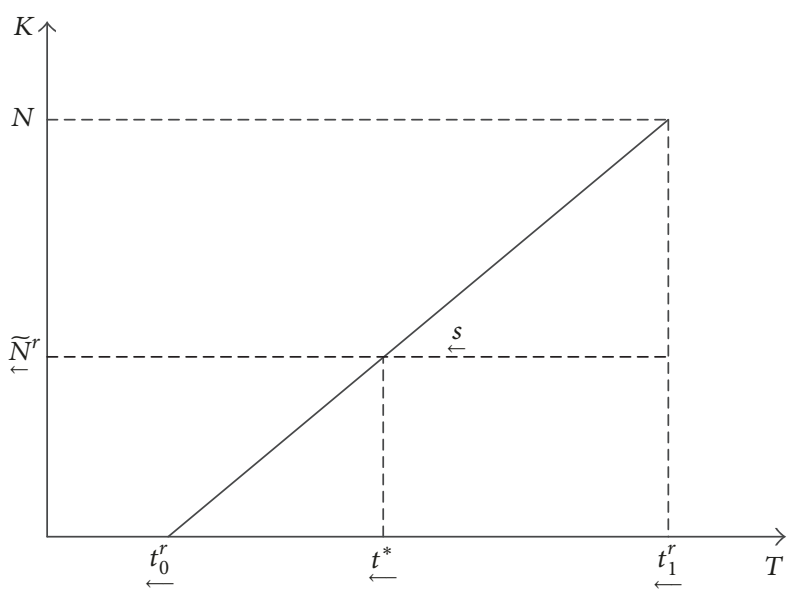

FIGURE 4: User equilibrium in evening commuting of regime $r$.

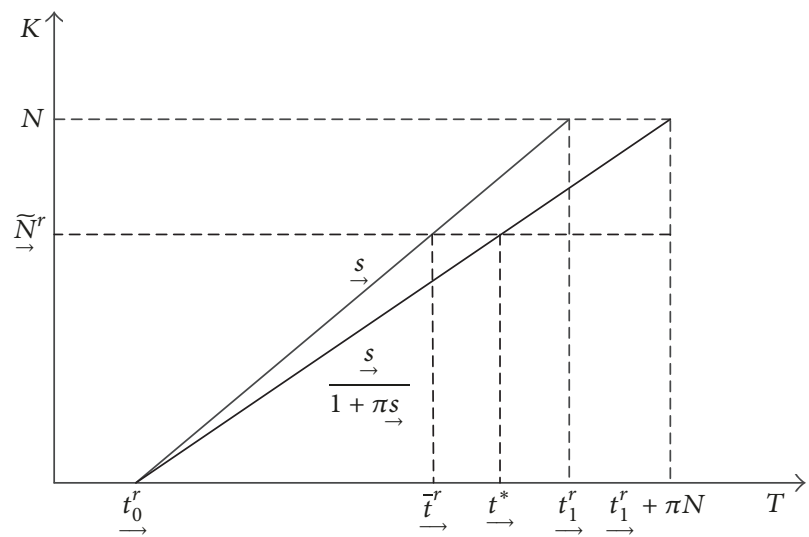

FIGURE 5: User equilibrium in morning commuting of regime $r$.

\section{Optimal Time-Varying Road \\ Tolls with Duration Dependent Parking Fees (Regime $o$ )}

In regime $o$, commuters need to pay for the road tolls and duration dependent parking fees in day-long commuting. One aim of time-varying road tolls is to eliminate the queue delay at bottleneck and another aim of time-varying road tolls is to minimize the total schedule delay cost and get the optimal starting and ending time of morning and evening commutes.

In Section 3, we have found that commuters are willing to delay the arrival time at workplace in morning commuting and putting forward departing time from workplace in evening commuting reduces duration dependent parking fees in regime $u$. So, in regime $u$, the total schedule delay cost is not optimal. Then, in regime $o$, we can determine the optimal rush hour interval by time-varying road tolls to achieve system optimum. However, the system cost in daylong commuting in regime $o$ cannot be reduced further on the basis of the system cost in regime $r$, because the queue delay is eliminated by road toll and the two regimes have the same and optimal total schedule delay costs.

Using an analytic method shown in Appendix C, we can obtain the optimal time-varying road toll function in evening commuting which can be given as

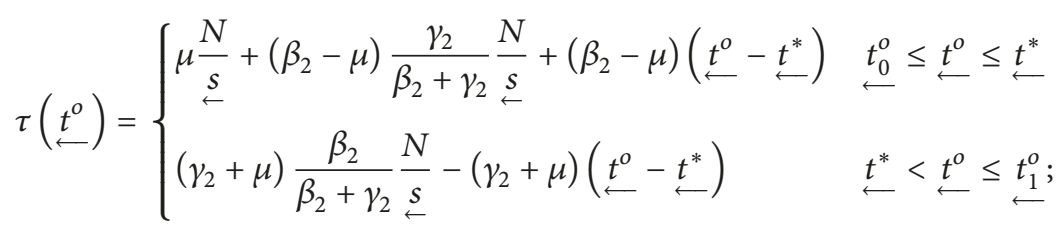


the optimal starting time and ending time of rush hour in evening commuting are

$$
\begin{aligned}
& t_{0}^{o}=t^{*}-\frac{\gamma_{2}}{\beta_{2}+\gamma_{2}} \frac{N}{s}, \\
& \stackrel{t_{1}^{o}}{\longleftarrow} t^{*}+\frac{\beta_{2}}{\beta_{2}+\gamma_{2}} \frac{N}{\leftarrow} .
\end{aligned}
$$

The individual travel cost in evening commuting of regime $o$ is

$$
C_{e}^{o}=\frac{\beta_{2} \gamma_{2}}{\beta_{2}+\gamma_{2}} \frac{N}{\leftarrow}+\frac{\beta_{2} \mu}{\beta_{2}+\gamma_{2}} \underset{\leftarrow}{\frac{N}{s}}+\mu\left(t^{t^{*}}-t^{\Delta}\right) .
$$

The system cost in evening commuting of regime $o$ is

$$
\mathrm{SC}_{e}^{o}=\frac{1}{2} \frac{\beta_{2} \gamma_{2}}{\beta_{2}+\gamma_{2}} \frac{N^{2}}{\stackrel{s}{\leftarrow}}
$$

Similarly, the optimal time-varying road toll in morning commuting can be obtained as follows:

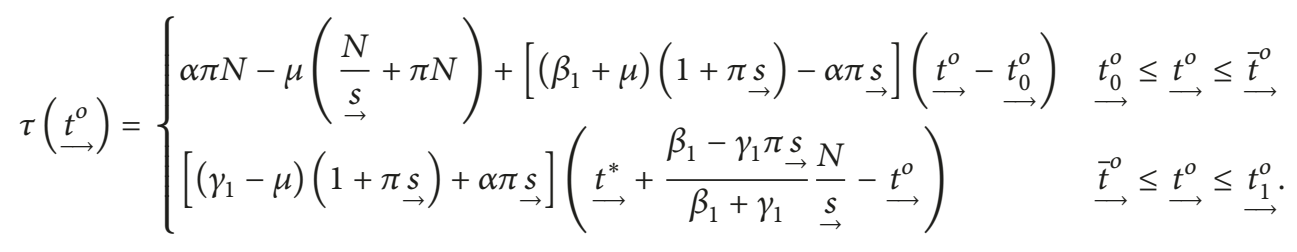

The optimal beginning time and ending time of rush hour in morning commuting are

$$
\begin{aligned}
& \stackrel{t_{0}^{o}}{\rightarrow} \stackrel{t^{*}}{\rightarrow}-\frac{\gamma_{1}(1+\pi \stackrel{s}{\rightarrow})}{\beta_{1}+\gamma_{1}} \frac{N}{s}, \\
& \stackrel{t_{1}^{o}}{\rightarrow}=\stackrel{t^{*}}{\rightarrow}+\frac{\beta_{1}-\gamma_{1} \pi \stackrel{s}{\rightarrow}}{\beta_{1}+\gamma_{1}} \underset{s}{\stackrel{N}{\rightarrow}} .
\end{aligned}
$$

The individual travel cost in morning commuting of regime $o$ is

$$
\begin{aligned}
C_{m}^{o}= & \frac{\beta_{1} \gamma_{1}(1+\pi \stackrel{s}{\rightarrow})}{\beta_{1}+\gamma_{1}} \frac{N}{\stackrel{s}{\rightarrow}}+\alpha \pi N+\mu\left(t^{\Delta}-\underline{t^{*}}\right) \\
& -\frac{\mu \beta_{1}(1+\pi \stackrel{s}{\rightarrow})}{\beta_{1}+\gamma_{1}} \frac{N}{\stackrel{s}{\rightarrow}} .
\end{aligned}
$$

The system cost in morning commuting of regime $o$ is

$$
\mathrm{SC}_{m}^{o}=\frac{1}{2} \alpha \pi N^{2}+\frac{1}{2} \frac{\beta_{1} \gamma_{1}}{\beta_{1}+\gamma_{1}} \frac{N^{2}}{\stackrel{s}{\rightarrow}}(1+\pi \underset{\rightarrow}{s}) .
$$

\section{Elastic Travel Demand considering Daily Travel Cost}

In this section, we investigate the efficiency of each pricing charge scheme proposed from Sections 2 to 5 in the elastic travel demand. The demand function for travel is given as follows:

$$
N=D(P), \quad \frac{d D}{d P}<0,
$$

where $N$ is the number of commuters and $P$ is the private daily travel cost. We assume the demand function $D(P)$ is a strictly decreasing linear function with respect to $P$. Let $D^{-1}(N)$ be the inverse demand function.

A commuter's surplus with travel demand $N$ is

$$
\operatorname{CS}(N)=\int_{0}^{N} D^{-1}(x) d x-\operatorname{ATC}(N) N,
$$

where the first term is the total gross benefit and the second term is the total travel cost. The total travel cost is

$$
\operatorname{ATC}(N) N=\operatorname{SC}(N)+R(N)
$$

where the first term is the total social cost and the second term is the total revenue. Then, we can obtain the social surplus $\mathrm{SS}(N)$ which is the sum of the commuter's surplus, the total revenue, and total externality

$$
\mathrm{SS}(N)=\int_{0}^{N} D^{-1}(x) d x-\operatorname{SC}(N),
$$

where $\operatorname{SS}(N)$ is the function of travel demand $N$.

Maximizing the social surplus leads to the following optimality condition:

$$
D^{-1}(N)=\operatorname{MSC}(N)
$$

where $\operatorname{MSC}(N)$ represents the marginal social cost.

Now we can apply our elastic demand case to evaluate four different parking pricing regimes proposed from Sections 2 to 5 . We first formulate the linear daily individual travel cost, $\operatorname{ATC}^{k}(N)=I^{k}+K^{k} N$ for regime $k, k=f, u, r, o$. $I^{f}=I^{r}=0, I^{u}=I^{o}=\mu\left(t^{*}-\underline{t^{*}}\right)$. The formulas of parameter $K^{k}$ in four regimes are given in Appendix D.

In market equilibrium, the implemented demand $N_{e}^{k}$ can be obtained by solving the two equations representing demand function $N=D(P)$ and cost function $P=I^{k}+K^{k} N$, respectively. And the total social cost is proportional to the 


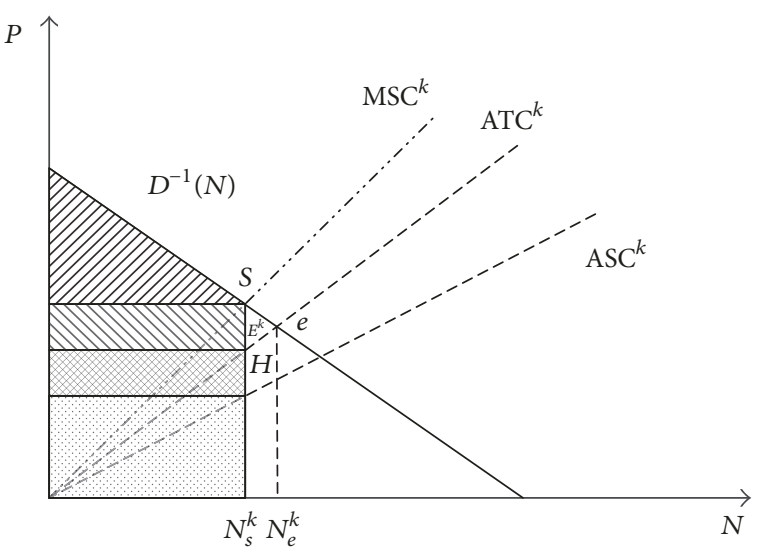

ZID Commuter's surplus Total revenue MIV Total externality $\quad$ Total social cost

FIGURE 6: Demand and supply function of day-long commuting.

square of the demand, $\operatorname{SC}^{k}(N)=L^{k} N^{2}$. The average social cost $\operatorname{ASC}^{k}(N)$ is $L^{k} N, \operatorname{ASC}^{k}(N)=L^{k} N$.

The marginal social cost becomes $\operatorname{MSC}^{k}(N)=2 L^{k} N$ and the optimal demand $N_{s}^{k}$ can be obtained by solving the demand function $N=D(P)$ and cost function $P=2 L^{k} N$. If the equilibrium demand $N_{e}^{k}$ is not less than the optimal demand $N_{s}^{k}, N_{e}^{k} \geq N_{s}^{k}$, to derive the travel demand $N_{e}^{k}$ to $N_{s}^{k}$, the commuter must be charged to internalize the externality $E^{k}$ which is the difference between marginal social cost and individual travel cost in pricing regime $k$. The externality $E^{k}$ is

$$
\begin{aligned}
E^{k} & =\operatorname{MSC}^{k}\left(N_{s}^{k}\right)-\operatorname{ATC}\left(N_{s}^{k}\right) \\
& =\left(2 L^{k}-K^{k}\right) N_{s}^{k}-I^{k},
\end{aligned}
$$

where $N_{s}^{k}$ is the optimal demand level in regime $k$.

If the equilibrium demand $N_{e}^{k}$ is less than the optimal demand $N_{s}^{k}, N_{e}^{k}<N_{s}^{k}$, to achieve system optimum, the commuter should be subsidized the negative externality $E^{k}$.

Next, we could compare the number of optimal travel demand $N_{s}^{k}$ in four regimes by determining the sequence of parameter $L^{k}$ at first. The process of comparing parameter $L^{k}$ is given in Appendix D.

In Figure $6, D^{-1}(N)$ is the reverse demand function. $\mathrm{MSC}^{k}$ represents marginal social cost and $\mathrm{ATC}^{k}$ is the daily individual travel cost function. ASC $^{k}$ is the average social cost. When there is no constant toll, the equilibrium appears at point $e$. Maximizing the social surplus can determine the optimal demand $N_{s}^{k}$ shown at the point $s$. SH represents the externality for each commuter; it can be charged by a constant road toll or parking fee to induce system optimum. At the optimal demand level, we present the constitution of commuter's surplus, total externality, total revenue, and total social cost. Here, total revenue is comprised of time-varying road toll and duration dependent parking fees. In regime $f$,

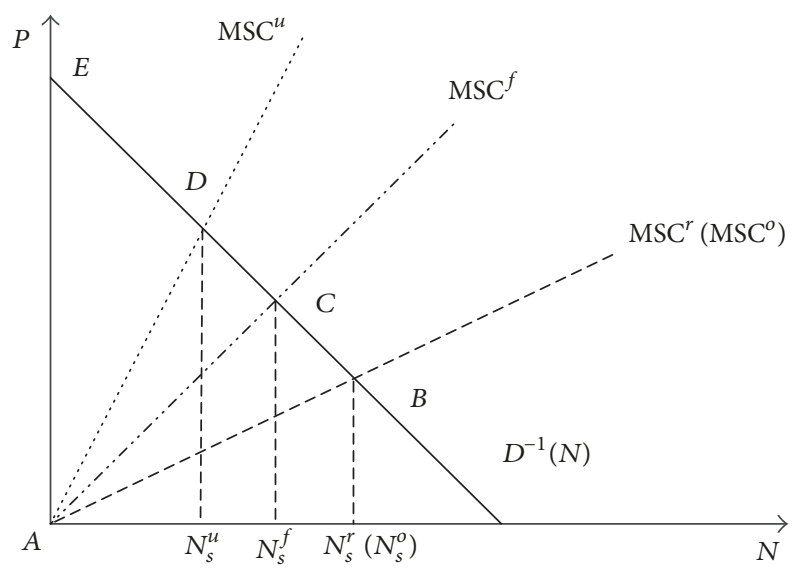

FIGURE 7: Comparisons of optimal travel demand and total social surplus in four regimes.

because of no road toll and parking fee, curve ASC and curve ATC are overlapped and the total revenue is zero.

In the case of elastic demand, we denote $N_{s}^{o}, N_{s}^{r}, N_{s}^{f}, N_{s}^{u}$ as the optimal travel demand in regimes $o, r, f, u$. Let $S S^{o}\left(N_{s}^{o}\right)$, $\mathrm{SS}^{r}\left(N_{s}^{r}\right), \mathrm{SS}^{f}\left(N_{s}^{f}\right), \mathrm{SS}^{u}\left(N_{s}^{u}\right)$ be the social surplus in regimes $o, r, f, u$. For the same demand function, we can easily find that $N_{s}^{o}=N_{s}^{r}>N_{s}^{f} \geq N_{s}^{u}$. In Figure 7, $\operatorname{SS}^{o}\left(N_{s}^{o}\right)$ and $\operatorname{SS}^{r}\left(N_{s}^{r}\right)$ are area $\mathrm{ABE}, \mathrm{SS}^{f}\left(N_{s}^{f}\right)$ is area $\mathrm{ACE}$, and $\mathrm{SS}^{u}\left(N_{s}^{u}\right)$ is area $\mathrm{ADE}$, so we can easily obtain that $\mathrm{SS}^{o}\left(N_{s}^{o}\right)=\operatorname{SS}^{r}\left(N_{s}^{r}\right)>\operatorname{SS}^{f}\left(N_{s}^{f}\right) \geq$ $\mathrm{SS}^{u}\left(N_{s}^{u}\right)$. When the parking fee rate $\mu$ is zero, $\mu=0$, the social surplus of regime $u \mathrm{SS}^{u}\left(N_{s}^{u}\right)$ is equivalent to that of regime $f \mathrm{SS}^{f}\left(N_{s}^{f}\right)$. If we set a positive parking fee rate $\mu, \mu>0$, the social efficiency of regime $u$ is less than that of regime $f$, $\operatorname{SS}^{u}\left(N_{s}^{u}\right)<\mathrm{SS}^{f}\left(N_{s}^{f}\right)$. We find that the best pricing regimes are regimes $o$ and $r$, followed by regime $f$; and the worst regime is regime $u$.

\section{Numerical Examples}

In the network shown in Figure 1, the service rate of each bottleneck is assumed to be $s=s=500 \mathrm{veh} / \mathrm{h}$ and the total number of commuters $N=1000$; they go to workplace from home in the morning and go back to home in the evening. We assume each commuter drives his/her car to workplace in the morning and returns home in the evening. The desired arrival time to workplace in the morning $\underset{t^{*}}{\longrightarrow}=9: 00$ and the desired departing time from workplace $t^{*}=17: 00$. The unit cost of travel time is $\alpha=10 \$ / \mathrm{h}$, the cost of unit early arrival time in the morning is $\beta_{1}=5 \$ / \mathrm{h}$, and the cost of unit late arrival time in the morning is $\gamma_{1}=20 \$ / \mathrm{h}$.

In evening commuting, the cost of unit early departing time is $\beta_{2}=20 \$ / \mathrm{h}$ and the cost of unit late departing time is $\gamma_{2}=5 \$ / \mathrm{h}$. And the searching time of unit parking spot $\pi$ is $0.72 \mathrm{~s} / \mathrm{spot}$. In regime $o$, the parking fee rate $\mu$ is $0.5 \$ / \mathrm{h}$ and, in regime $u$, we set parking fee rate from $1 \$ / \mathrm{h}$ to $4 \$ / \mathrm{h}$. The demand function is specified as $N(P)=2000-20 P$, where $P$ is the daily private travel cost. 
TABLE 1: Main computing results of various regimes in day-long commuting with elastic demand.

\begin{tabular}{|c|c|c|c|c|c|c|c|c|c|c|}
\hline \multirow{2}{*}{ Regime } & \multirow{2}{*}{$r$} & \multirow{2}{*}{$o$} & \multirow{2}{*}{$f$} & \multicolumn{7}{|c|}{$u$} \\
\hline & & & & 1 & 1.5 & 2 & 2.5 & 3 & 3.5 & 4 \\
\hline Equilibrium demand & 1453 & 1384 & 1488 & 1321 & 1244 & 1171 & 1101 & 1034 & 970 & 908 \\
\hline Optimal demand & 1453 & 1453 & 1185 & 1139 & 1116 & 1093 & 1072 & 1050 & 1029 & 1009 \\
\hline Externality & 0 & -4.84 & 20.38 & 12.72 & 9.10 & 5.57 & 2.15 & -1.17 & -4.42 & -7.60 \\
\hline Individual travel cost & 27.33 & 32.17 & 20.38 & 30.35 & 35.12 & 39.76 & 44.27 & 48.67 & 52.95 & 57.14 \\
\hline Social surplus $\left(10^{3}\right)$ & 72.674 & 72.674 & 59.242 & 56.927 & 55.789 & 54.671 & 53.576 & 52.507 & 51.468 & 50.460 \\
\hline Marginal social cost & 27.33 & 27.33 & 40.76 & 43.07 & 44.21 & 45.33 & 46.42 & 47.49 & 48.53 & 49.54 \\
\hline Social cost $\left(10^{3}\right)$ & 19.859 & 19.859 & 24.146 & 24.520 & 24.665 & 24.782 & 24.872 & 24.937 & 24.978 & 24.998 \\
\hline Revenue $\left(10^{3}\right)$ & 19.859 & 26.897 & 0 & 10.036 & 14.523 & 18.690 & 22.564 & 26.170 & 29.531 & 32.669 \\
\hline
\end{tabular}

TABLE 2: Main computing results in regime $k(k=f, r, o, u)$ in morning commuting under optimal demand.

\begin{tabular}{|c|c|c|c|c|c|c|c|c|c|c|}
\hline \multirow{2}{*}{ Regime $k(k=f, r, o, u)$} & \multirow{2}{*}{$r$} & \multirow{2}{*}{$o$} & \multirow{2}{*}{$f$} & \multicolumn{7}{|c|}{$u$ with the following parking fee rate } \\
\hline & & & & 1 & 1.5 & 2 & 2.5 & 3 & 3.5 & 4 \\
\hline$\stackrel{r_{1}^{k}}{\longrightarrow}$ & 500 & 500 & 909 & 1136 & 1299 & 1515 & 1818 & 2273 & 3030 & 4545 \\
\hline$\stackrel{r_{2}^{k}}{\longrightarrow}$ & 500 & 500 & 152 & 157 & 159 & 162 & 165 & 168 & 172 & 175 \\
\hline$\tilde{N}^{n}$ & 1163 & 1163 & 991 & 907 & 866 & 827 & 789 & 752 & 717 & 683 \\
\hline$\delta_{\rightarrow}^{k}$ & 0.80 & 0.80 & 0.84 & 0.8 & 0.78 & 0.76 & 0.74 & 0.72 & 0.70 & 0.68 \\
\hline$t_{0}^{k}$ & $6: 26$ & $6: 26$ & $6: 49$ & $7: 00$ & $7: 05$ & $7: 10$ & $7: 15$ & $7: 21$ & $7: 25$ & $7: 30$ \\
\hline$\stackrel{\overrightarrow{t_{1}^{k}}}{\longrightarrow}$ & $9: 21$ & $9: 21$ & $9: 11$ & $9: 17$ & $9: 20$ & $9: 22$ & $9: 24$ & $9: 27$ & $9: 29$ & 9:31 \\
\hline
\end{tabular}

Table 1 gives the main computing results. We can find the regimes $r$ and $o$ have the highest social surplus. In regime $r$, the externality is zero and it means that the time-vary road toll can automatically lead to the system optimum. In regime $o$, the equilibrium demand is less than the optimal demand and the externality is negative; the decision maker needs to subsidize commuters to achieve system optimum. In regime $u$, when parking fee rate $\mu$ is less than $2.822 \$ / \mathrm{h}$, the externality is charged to balance the optimal demand and supply. Otherwise, when parking fee rate is greater than $2.822 \$ / \mathrm{h}$, the commuters should be subsidized to achieve system optimum due to the negative externality. Besides, we also find that the social surplus decreases as the parking fee rate increases in regime $u$ and the parking fee has a negative social effect. It means regime $u$ has the highest social surplus when the parking fee rate is zero.

In Table 2, $r_{1}^{k}$ is the departing rate of early arrival commuter to workplace and $r_{2}^{k}$ is the departing rate of late arrival commuter to workplace in regime $k, k=\{r, o, f, u\}$. The number of early arrival commuters in regime $k$ in morning commuting is $\underset{\sim}{\stackrel{N}{N}} \cdot t_{0}^{k}$ is the departure time of the first commuters and $t_{1}^{k}$ is the departure time of the last commuter in regime $k$.

In morning commuting, the departure rates from home of early arrival and late arrival of regimes $r$ and $o$ are equivalent to the capacity of bottleneck due to the time-varying road toll. In regimes $r$ and $o$, the optimal starting time is $6: 26$ and

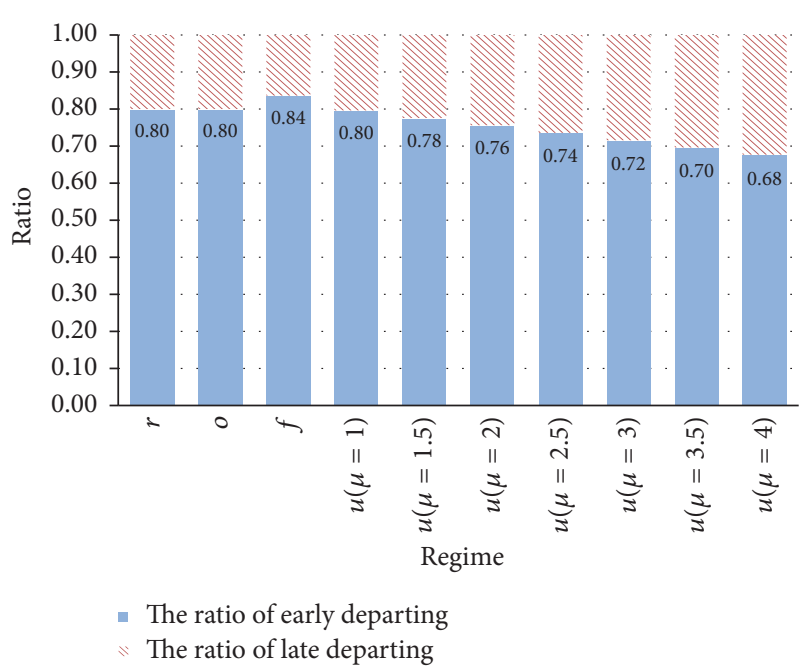

FIgURE 8: The ratio of early and late departing commuters to total demand of four pricing regimes in the morning commuting.

the optimal ending time is 9:21. In regime $u$, as the duration dependent parking fee rate $\mu$ increases, the departure rates from home $r_{1}^{u}$ and $r_{2}^{u}$ increase and the commuters push back their starting and ending time of the morning commuting, $t_{0}^{u}$ and $t_{1}^{u}$ to reduce the duration dependent parking fee. $\overrightarrow{\text { Figure }} 8 \overrightarrow{\mathrm{sh}}$ ows the ratio of early departing commuters of four schemes. When the parking fee rate $\mu$ increases from $0 \$ /$ h to $4 \$ / h$, the ratio of the number of early departing commuters 
TABLE 3: Main computing results in regime $k(k=f, r, o, u)$ in evening commuting under optimal demand.

\begin{tabular}{|c|c|c|c|c|c|c|c|c|c|c|}
\hline \multirow{2}{*}{ Regime $k(k=f, r, o, u)$} & \multirow{2}{*}{$r$} & \multirow{2}{*}{$o$} & \multirow{2}{*}{$f$} & \multicolumn{7}{|c|}{$u$ with the following parking fee rate $\mu$} \\
\hline & & & & 1 & 1.5 & 2 & 2.5 & 3 & 3.5 & 4 \\
\hline$r_{1}^{k}$ & 500 & 500 & 1500 & 1450 & 1425 & 1400 & 1375 & 1350 & 1325 & 1300 \\
\hline$\stackrel{r_{2}^{k}}{\longleftarrow}$ & 500 & 500 & 250 & 200 & 175 & 150 & 125 & 100 & 75 & 50 \\
\hline $\begin{array}{l}\overleftarrow{\sim}^{k} \\
\stackrel{N}{\longleftarrow}\end{array}$ & 291 & 291 & 711 & 792 & 827 & 857 & 884 & 907 & 927 & 945 \\
\hline$\delta_{\leftarrow}^{k}$ & 0.20 & 0.20 & 0.60 & 0.70 & 0.74 & 0.78 & 0.82 & 0.86 & 0.90 & 0.94 \\
\hline$\stackrel{t_{0}^{k}}{\longleftarrow}$ & $16: 25$ & $16: 25$ & $16: 32$ & $16: 27$ & $16: 25$ & $16: 23$ & $16: 21$ & $16: 20$ & $16: 18$ & $16: 16$ \\
\hline$\stackrel{t}{t_{1}^{k}}$ & $19: 20$ & $19: 20$ & $18: 53$ & $18: 44$ & $18: 39$ & $18: 34$ & $18: 30$ & $18: 26$ & $18: 22$ & $18: 18$ \\
\hline
\end{tabular}

to total demand $\delta^{k}$ decreases from 0.84 to 0.68 . It means that more commuters depart late from home as the parking fee rate increases in the morning commuting of regime $u$.

Table 3 lists the main numerical results in regime $k$, $k=\{r, o, f, u\}$ in the evening commuting. Here, $r_{1}^{k}$ is the departing rate of early departing commuters from workplace and $r_{2}^{k}$ is the departing rate of late departing commuters from workplace. The number of early departing commuters in evening commuting is $\tilde{\sim}^{k} \cdot \underbrace{k}_{0}$ is the departure time of the first commuter and $t_{1}^{k}$ is the departure time of the last commuter.

In the evening commuting of regimes $r$ and $o$, the queues are eliminated by the road toll, so the departure rates from workplace of early departing commuters and late departing commuters $r_{1}^{k}, r_{2}^{k}, k=r, o$, are same to the capacity of bottleneck $s$. In regimes $r$ and $o$, the optimal starting time of rush time $t_{0}^{r}, t_{0}^{o}$ is 16:25 and the optimal ending time $t_{1}^{r}$, $t_{1}^{o}$ is 19:20. In regime $u$, as the duration dependent parking $\overleftarrow{\text { fee }}$ rate $\mu$ increases, the departure rates from workplace $r_{1}^{u}$, $r_{2}^{u}$ are reduced, and the commuters put forward their starting and ending time of the evening commuting $t_{0}^{u}, t_{1}^{u}$ to reduce the duration dependent parking fee. Figure 9 shows the ratio of early departing commuters of four schemes in the evening commuting. When the parking fee rate $\mu$ increases from $0 \$ / \mathrm{h}$ to $4 \$ / h$, the ratio of the number of early departing commuters to total demand $\delta^{k}$ also increases from 0.6 to 0.94 . It means that more commuters depart from workplace early with the growth of parking fee rate in the evening commuting of regime $u$.

Figures 10 and 11 show the road toll curves of evening and morning commutes in regimes $r$ and $o$. The two pricing schemes have the same and optimal starting time and ending time in evening and morning commutes. In the evening commuting, the optimal starting and ending times of rush time of regimes $r$ and $o$ are 16:25 and 19:20. The road tolls of first commuter and last commuter are zero in regime $r$.

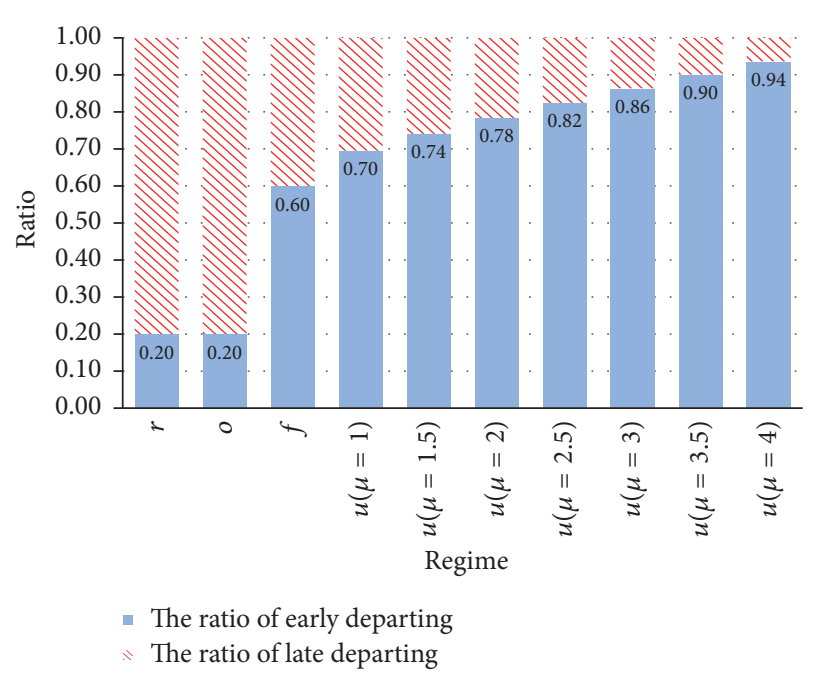

FIGURE 9: The ratio of early and late departing commuters to total demand of four pricing regimes in the evening commuting.

The road toll of first commuter in regime $o$ is $1.45 \$$ and the road toll of last commuter is zero. The commuter who departs from workplace at 17:00 should pay for the maximum road toll $12.79 \$$ in regime $o$ and the maximum road toll $11.62 \$$ in regime $r$.

In the morning commuting, the optimal starting and ending times of rush time are 6:26 and 9:21. In regime $r$, the road toll of first commuter is $2.91 \$$ and the road toll of last commuter is zero. In regime $o$, the road toll of first commuter is $1.31 \$$ and the road toll of last commuter is zero. The commuter who arrives at workplace on time should pay for the maximum road toll $13.05 \$$ in regime $o$ and the maximum road toll $13.37 \$$ in regime $r$.

\section{Conclusions}

In this paper, we extend the bottleneck model from a single morning commuting to a day-long commuting. The morning commuting and evening commuting are treated as two independent user equilibriums. On the basis of previous study of Zhang et al. [23], we relax the assumption by allowing commuters to arrive later than the desired arrival time in 


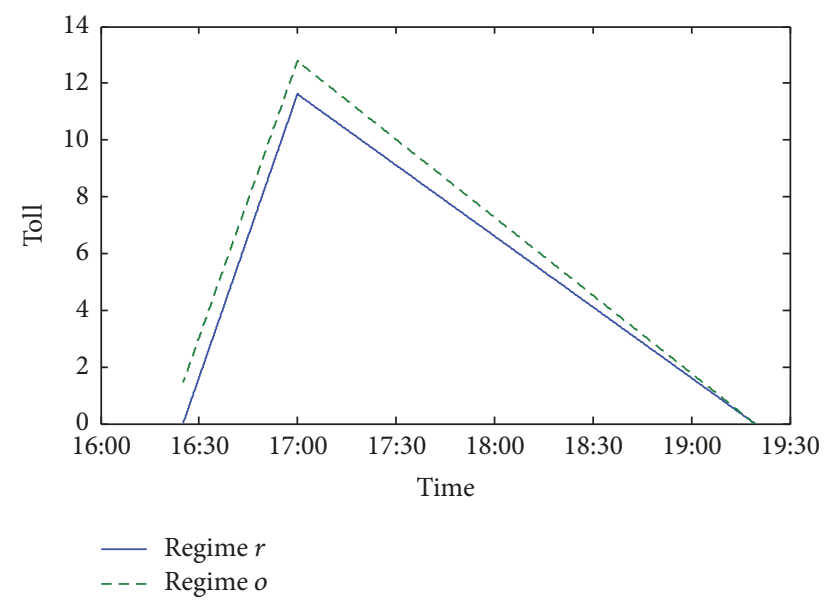

FIGURE 10: Time-varying road toll of evening commuting in regimes $r$ and $o$.

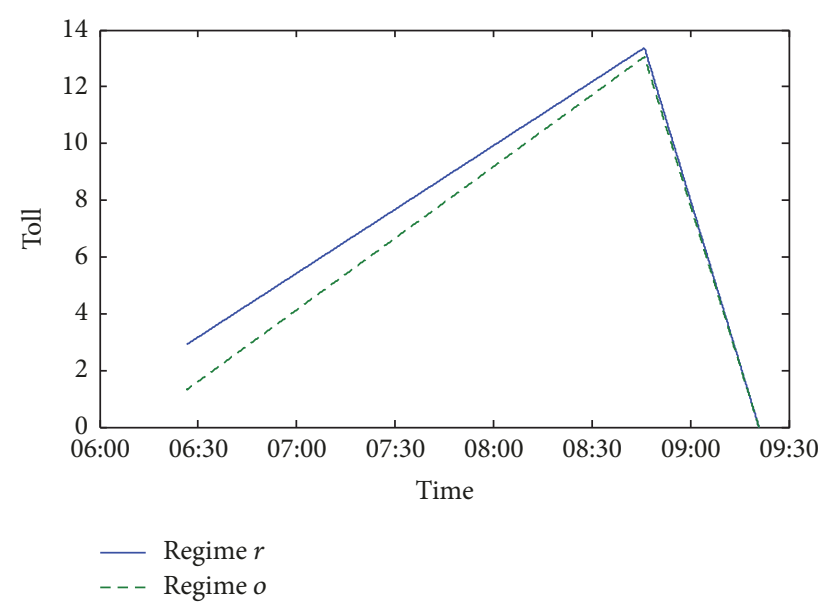

FIGURE 11: Time-varying road toll of morning commuting in regimes $r$ and $o$.

morning commuting and depart earlier than the desired time in evening commuting. Besides, we assume that the morning and evening commutes are symmetric.

We investigated the day-long commuting of four regimes (regimes $f, u, r, o$ ) and derived the traffic pattern of each regime. In user equilibrium of regime $f$ and $u$, no one can reduce the individual travel cost by changing the departure time. In regimes $r$ and $o$, the queues at the bottleneck of morning and evening commutes are eliminated by road toll. We first determine the optimal starting and ending time of rush hour by minimizing the total schedule delay cost. Then, we utilize road toll to achieve system optimum.

Next, we analyze efficiency of four schemes with elastic demand and find that the regimes $r$ and $o$ have the highest social surplus, followed by regime $f$ and the regime $u$ has the lowest social surplus. In regime $u$, the duration parking fee may drive commuters to narrow their work duration for reducing the parking fee and the duration parking fee cannot increase social surplus. Furthermore, as the parking fee rate increases, the social surplus decreases. In regimes $r$ and $o$, the optimal travel demands are equivalent and the two pricing schemes have the highest social surplus. However, in regime $o$, the duration dependent parking fee should be subsidized to commuters to balance the optimal travel demand and supply as a result of negative externality.

These findings also have several implications for traffic management; first, the duration dependent parking fee may reduce the average parking time per car to relieve the pressure of parking demand, but it will reduce the social surplus under optimal travel demand. Second, the time-varying road toll can eliminate the queue delay and minimize schedule delay cost by optimizing the arrival and departure time interval in morning and evening commutes in regime $r$ and $o$. Third, if the time-varying road toll is carried out, it is inefficient to charge duration dependent parking fee; the duration dependent parking fee cannot increase the social surplus in basis of time-varying road toll except for refunding a part of revenue as a subsidy to commuters.

The current paper considers the morning commuting and evening commuting are symmetric. However, the symmetry between morning and evening commutes is easily broken when commuters are heterogeneous [20]. If the commutes have different desired working starting and ending times, then the traffic pattern could be FIFO (first in first out) in daylong commuting and the morning and evening commutes may not be independent. So if we relax this assumption, the single morning or evening commuting is not an independent user equilibrium anymore, and the problem may be more complicated and worth investigating in the future.

\section{Appendix}

\section{A. User Equilibrium in Duration Dependent Parking Fees}

In regime $u$, the individual travel cost of the commuter who leaves workplace early in evening commuting is

$$
\begin{aligned}
C_{e}^{u}\left(t^{u}, \mu\right)= & \alpha \frac{D\left(\stackrel{t^{u}}{\longleftarrow}\right)}{s}+\beta_{2}\left(t^{t^{*}}-t^{u}\right) \\
& +\mu\left(\stackrel{t^{u}}{\longleftarrow}-t^{\Delta}\right) .
\end{aligned}
$$

The individual travel cost of the commuter who leaves workplace late is

$$
\begin{aligned}
C_{e}^{u}\left(\stackrel{t^{u}}{\longleftarrow}, \mu\right)= & \alpha \frac{D\left(\stackrel{t^{u}}{\longleftarrow}\right)}{\stackrel{s}{\longleftarrow}}+\gamma_{2}\left(t^{t^{u}}-t^{*}\right) \\
& +\mu\left(\stackrel{t^{u}}{\longleftarrow}-t^{\Delta}\right) .
\end{aligned}
$$

According to the equilibrium condition $\partial C_{e}^{u} / \partial t^{u}=0$, we can get

$$
\frac{d D\left(\frac{\left.t^{u}\right)}{\leftarrow}\right)}{d t^{u}}= \begin{cases}\stackrel{s}{\longleftarrow}\left(\beta_{2}-\mu\right) & t_{0}^{u} \leq t^{u} \leq t^{*} \\ -\frac{s}{\alpha}\left(\gamma_{2}+\mu\right) & \stackrel{t^{*}}{\longleftarrow}<t^{t^{u}} \leq t_{1}^{u} .\end{cases}
$$


From (A.3), the departure rate from workplace $r_{1}^{u}(\mu), r_{2}^{u}(\mu)$ could be solved, $r_{1}^{u}(\mu)=s\left(1+\left(\beta_{2}-\mu\right) / \alpha\right), r_{2}^{u}(\mu)=\underbrace{}_{\llcorner}\left(1-\left(\gamma_{2}+\right.\right.$ $\mu) / \alpha$ ). Here, we require the parking fee rate $\mu$ not to exceed $\beta_{2}, \mu<\beta_{2}$, to ensure the growing queue at bottleneck. Then, in regime $u$, we have $r_{1}^{u}(\mu)>s, r_{2}^{u}(\mu)<s$.

The equilibrium requires the travel costs of the first and last commuter to be equivalent, $C_{e}^{u}\left(t_{0}^{u}, \mu\right)=C_{e}^{u}\left(t_{1}^{u}, \mu\right)$, which is presented in the equation as follows:

$$
\begin{aligned}
& \beta_{2}\left(\stackrel{t^{*}}{\longleftarrow}-t_{0}^{u}\right)+\mu\left(\stackrel{\left.t_{0}^{u}-t^{\Delta}\right)}{\longleftarrow}\right. \\
& \quad=\gamma_{2}\left(\stackrel{t_{1}^{u}-t^{*}}{\longleftarrow}\right)+\mu\left(t_{t_{1}^{u}}^{\longleftarrow}-t^{\Delta}\right) .
\end{aligned}
$$

And the length of evening commuting is $N / \stackrel{s}{\leftarrow}$, so the first commuter's departure time and the last commuter's departure times are

$$
\begin{aligned}
& t_{0}^{u}(\mu)=t^{*}-\left(\frac{\gamma_{2}+\mu}{\beta_{2}+\gamma_{2}}\right) \frac{N}{s}, \\
& t_{1}^{u}(\mu)=t^{*}+\left(\frac{\beta_{2}-\mu}{\beta_{2}+\gamma_{2}}\right) \frac{N}{s} .
\end{aligned}
$$

For morning commuting in regime $u$, when $t_{0}^{u} \leq \underline{t^{u}} \leq \underline{\bar{t}^{u}}$, the individual travel cost for the commuter who arrives to workplace early is

$$
\begin{aligned}
C_{m}^{u}\left(\stackrel{t^{u}}{\rightarrow}, \mu\right)= & \alpha\left[\frac{D\left(\underline{t^{u}}\right)}{s}+\pi \stackrel{s}{\rightarrow}\left(\stackrel{t^{u}}{\rightarrow}-\stackrel{t_{0}^{u}}{\rightarrow}\right)\right] \\
& +\beta_{1}\left[\stackrel{t^{*}}{\rightarrow}-\underline{t^{u}}-\pi \underset{s}{\rightarrow}\left(\stackrel{t^{u}}{\rightarrow}-\stackrel{t_{0}^{u}}{\rightarrow}\right)\right] \\
& +\mu\left[t^{\Delta}-\underline{t^{u}} \rightarrow \pi \stackrel{s}{\rightarrow}\left(\stackrel{t^{u}}{\rightarrow}-\stackrel{t_{0}^{u}}{\rightarrow}\right)\right] .
\end{aligned}
$$

On right-hand side of (A.6), the first term is the cost of travel time including queuing time and searching time for parking spots, the second term is the schedule delay cost for early arrival, and the last term is the parking fee.

When $\stackrel{\bar{t}^{u}}{\rightarrow} \leq \underline{t^{u}} \leq \underline{t_{1}^{u}}$, the individual travel cost for the commuter who arrives at workplace late is

$$
\begin{aligned}
C_{m}^{u}\left(\stackrel{t^{u}}{\rightarrow}, \mu\right)= & \alpha\left[\frac{D\left(\underline{t^{u}}\right)}{\stackrel{s}{\rightarrow}}+\pi \stackrel{s}{\rightarrow}\left(\underline{t^{u}}-\stackrel{t_{0}^{u}}{\rightarrow}\right)\right] \\
& +\gamma_{1}\left[\stackrel{t^{u}}{\rightarrow}+\pi \underset{\rightarrow}{\rightarrow}\left(\stackrel{t^{u}}{\rightarrow}-\stackrel{t_{0}^{u}}{\rightarrow}\right)-\underline{t^{*}} \rightarrow\right] \\
& +\mu\left[t^{\Delta}-\stackrel{t^{u}}{\rightarrow}-\pi \stackrel{s}{\rightarrow}\left(\stackrel{t^{u}}{\rightarrow} \stackrel{t_{0}^{u}}{\rightarrow}\right)\right] .
\end{aligned}
$$

According to the user equilibrium condition $\partial C_{m}^{u} / \partial \underline{t^{u}} \rightarrow=0$, we can get

$$
\begin{aligned}
& \frac{d D\left({\stackrel{t^{u}}{\rightarrow}}^{\rightarrow}\right)}{d \stackrel{t^{u}}{\rightarrow}}
\end{aligned}
$$

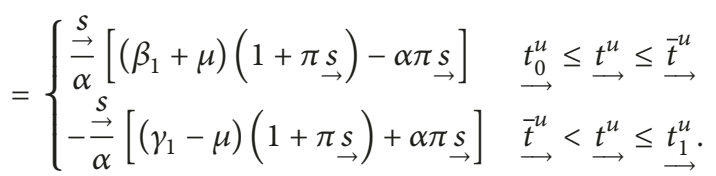

As the capacity of bottleneck is $\underset{\rightarrow}{\rightarrow}$ in morning commuting, the length of morning rush hour interval is $N / \vec{s}$. In equilibrium, the travel costs of the first commuter are equivalent to that of the last commuter:

$$
\begin{aligned}
\beta_{1}\left(\stackrel{t^{*}}{\rightarrow}-\stackrel{t_{0}^{u}}{\rightarrow}\right)+\mu\left(t^{\Delta}-\underline{t_{0}^{u}}\right) \\
=\gamma_{1}\left(\stackrel{t_{1}^{u}}{\longrightarrow}+\pi N-\stackrel{t^{*}}{\longrightarrow}\right)+\mu\left(t^{\Delta}-\stackrel{t_{1}^{u}}{\longrightarrow} \pi N\right) \\
\quad+\alpha \pi N .
\end{aligned}
$$

The arrival times to parking lot of the first and last commuter can be obtained:

$$
\begin{aligned}
& \stackrel{t_{0}^{u}}{\rightarrow}(\mu)=\stackrel{t^{*}}{\rightarrow}+\frac{\mu-\gamma_{1}}{\beta_{1}+\gamma_{1}} \frac{N}{\rightarrow}+\frac{\mu-\gamma_{1}}{\beta_{1}+\gamma_{1}} \pi N-\frac{\alpha \pi N}{\beta_{1}+\gamma_{1}}, \\
& \stackrel{t_{1}^{u}}{\rightarrow}(\mu)=\stackrel{t^{*}}{\rightarrow}+\frac{\beta_{1}+\mu}{\beta_{1}+\gamma_{1}} \frac{N}{\stackrel{s}{\rightarrow}}+\frac{\mu-\gamma_{1}}{\beta_{1}+\gamma_{1}} \pi N-\frac{\alpha \pi N}{\beta_{1}+\gamma_{1}} .
\end{aligned}
$$

\section{B. User Equilibrium in Optimal Time-Varying Road Tolls}

For evening commuting in regime $r$, the queue is eliminated by time-varying road toll. So the individual travel cost of the commuter who departs early from workplace is

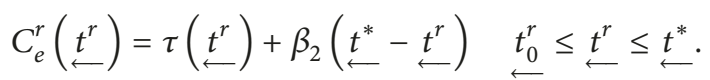

Here, $t^{r}$ is the departing time from workplace and $t_{0}^{r}$ is the departing time of the first commuter. On the right-hand side of (B.1), the first term is the road toll to the commuter with departing time $t^{r}$; the second term is the schedule delay cost for early departing.

The individual travel cost for the commuter who departs late from workplace is

$$
C_{e}^{r}\left(\stackrel{t}{t}^{r}\right)=\tau\left(t^{t^{r}}\right)+\gamma_{2}\left(\longleftarrow t^{r}-t^{*}\right) \quad t^{*}<t^{r} \leq t_{1}^{r} .
$$

User equilibrium condition $d C_{e}^{r} / d t^{r}=0$ requires

$$
\frac{d \tau\left(t^{r}\right)}{d t^{t^{r}}}= \begin{cases}\beta_{2} & \stackrel{t_{0}^{r} \leq t^{r} \leq t^{*}}{\longleftarrow} \\ -\gamma_{2} & \stackrel{t^{*}}{\longleftarrow}<t^{t^{r}} \leq t_{1}^{r} .\end{cases}
$$


The optimal rush hour interval is determined by minimizing the total schedule delay cost. Given the arrival rate $s$, total schedule delay cost is minimized by equating the schedule delay cost of the first and last commuters:

$$
\beta_{2}\left(\stackrel{t^{*}}{\longleftarrow} \underset{t}{t_{0}^{r}}\right)=\gamma_{2}\left(\stackrel{t_{1}^{r}}{\longleftarrow}-t^{*}\right)
$$

Combining (B.4) with the condition $t_{1}^{r}-t_{0}^{r}=N / \stackrel{s}{\leftarrow}$, we obtain

$$
\begin{aligned}
& \stackrel{t_{0}^{r}}{\longleftarrow} t^{*}-\frac{\gamma_{2}}{\beta_{2}+\gamma_{2}} \frac{N}{\leftarrow}, \\
& \stackrel{t_{1}^{r}}{\longleftarrow} t^{*}+\frac{\beta_{2}}{\beta_{2}+\gamma_{2}} \frac{N}{\longleftarrow} .
\end{aligned}
$$

To keep a lowest no-negative toll level, the last commuter pays no toll, $\tau\left(t_{1}^{r}\right)=0$. In the user equilibrium, the travel cost of the first commuter is equivalent to that of the last commuter:

$$
C_{e}^{r}\left(\begin{array}{c}
t_{0}^{r} \\
\longleftarrow
\end{array}\right)=C_{e}^{r}\left(\begin{array}{c}
t_{1}^{r} \\
\longleftarrow
\end{array}\right) .
$$

Given (B.1)-(B.3) and (B.5)-(B.6) and the user equilibrium condition, we can obtain the optimal time-varying road toll presented as follows:

$$
\begin{aligned}
& \tau\left(t^{r}\right) \\
& = \begin{cases}\frac{\beta_{2} \gamma_{2}}{\beta_{2}+\gamma_{2}} \frac{N}{s}+\beta_{2}\left(t^{r}-t^{*}\right) & t_{0}^{r} \leq t^{r} \leq t^{*} \\
\frac{\beta_{2} \gamma_{2}}{\beta_{2}+\gamma_{2}} \frac{\stackrel{N}{s}}{\longleftarrow}-\gamma_{2}\left(t^{t^{r}}-t^{t^{*}}\right) & \longleftarrow t^{*}<t^{r} \leq t_{1}^{r} .\end{cases}
\end{aligned}
$$

For the morning commuting in regime $r$, as the queue is eliminated by road toll, the travel time only is the searching time for parking spots. So the individual travel cost of commuter who arrive early is

$$
\begin{aligned}
C_{m}^{r}\left(\stackrel{t^{r}}{\rightarrow}\right)= & \alpha \pi \underset{s}{\rightarrow}\left(\stackrel{t^{r}}{\rightarrow}-\underline{t_{0}^{r}}\right) \\
& +\beta_{1}\left[\stackrel{t^{*}}{\rightarrow}-\underline{t^{r}}-\pi \stackrel{s}{\rightarrow}\left(\underline{t^{r}}-\underline{t_{0}^{r}}\right)\right] \\
& +\tau\left(\stackrel{t^{r}}{\longrightarrow}\right) \quad \stackrel{t_{0}^{r}}{\longrightarrow} \underline{t^{r}} \leq \underline{\bar{t}^{r}} .
\end{aligned}
$$

Here, $\underset{t^{r}}{\longrightarrow}$ is the arrival time to parking lot, $t_{0}^{r}$ is the arrival time to parking lot of the first commuter, and $\vec{t}^{r}$ is arrival time to parking lot of the commuter who arrives at workplace on time. On right-hand side of (B.8), the first term is the cost of searching time for parking spots, the second term is schedule delay cost for early arrival, and the third term is time-varying road toll.
The individual travel cost of the commuter who arrive late is

$$
\begin{aligned}
C_{m}^{r}\left(\stackrel{t^{r}}{\rightarrow}\right)= & \alpha \pi \underset{s}{\rightarrow}\left(\stackrel{t^{r}}{\rightarrow} \underset{t_{0}^{r}}{\rightarrow}\right) \\
& +\gamma_{1}\left[\stackrel{t^{r}}{\longrightarrow}+\underset{\pi}{\rightarrow}\left(\underline{t^{r}}-\underline{t_{0}^{r}}\right)-\underline{t^{*}} \rightarrow\right] \\
& +\tau\left(\stackrel{t^{r}}{\longrightarrow}\right) \stackrel{\bar{t}^{r}}{\longrightarrow}<\underline{t^{r}} \leq \underline{t_{1}^{r}} .
\end{aligned}
$$

According to the equilibrium condition $d C_{m}^{r} / d \underline{t^{r}}=0$, we can get

$$
\begin{aligned}
& \frac{d \tau\left(\underline{t^{r}}\right)}{d \stackrel{t^{r}}{\rightarrow}} \\
& \quad= \begin{cases}\beta_{1}(1+\pi \underset{s}{\rightarrow})-\alpha \pi \stackrel{s}{\rightarrow} & \underline{t_{0}^{r}} \leq \underline{t^{r}}<\underline{\bar{t}^{r}} \\
-\alpha \pi \underset{s}{\rightarrow}-\gamma_{1}(1+\pi \underset{s}{\rightarrow}) & \underline{\bar{t}^{r}}<\underline{t^{r}} \leq \underline{t_{1}^{r}} .\end{cases}
\end{aligned}
$$

The arrival rate to parking lot is given as $\underset{s}{\rightarrow}$ by optimal timevarying road toll, so the length of rush hour interval is $\mathrm{N} / \stackrel{\mathrm{s}}{\rightarrow}$. For the morning commuting, the optimal rush hour interval is determined by minimizing the total schedule delay cost. The beginning and ending time of rush hour are obtained by

$$
\beta_{1}\left(\stackrel{t^{*}}{\longrightarrow} \underset{t_{0}^{r}}{\longrightarrow}\right)=\gamma_{1}\left(\stackrel{t_{1}^{r}}{\longrightarrow}+\pi N-\underline{t^{*}}\right) .
$$

According to the length of interval and (B.11), we can get

$$
\begin{aligned}
& \stackrel{t_{0}^{r}}{\rightarrow} \stackrel{t^{*}}{\rightarrow}-\frac{\gamma_{1}(1+\pi \stackrel{s}{\rightarrow})}{\beta_{1}+\gamma_{1}} \frac{N}{s}, \\
& \stackrel{t_{1}^{r}}{\rightarrow}=\underline{t^{*}}+\frac{\beta_{1}-\gamma_{1} \pi \stackrel{s}{\rightarrow} \frac{N}{\beta_{1}+\gamma_{1}}}{\stackrel{s}{\rightarrow}}, \\
& \stackrel{\vec{t}^{r}}{\rightarrow}=\underline{t^{*}}-\frac{\gamma_{1} \pi \stackrel{s}{\rightarrow}}{\beta_{1}+\gamma_{1}} \stackrel{N}{\rightarrow} .
\end{aligned}
$$

To keep a lowest nonnegative toll level, the road toll of last commuter is set to zero, $\tau\left(t_{1}^{r}\right)=0$. In user equilibrium, the travel costs of the first and last commuter are equivalent:

$$
C_{m}^{r}\left(\stackrel{t_{0}^{r}}{\longrightarrow}\right)=C_{m}^{r}\left(\stackrel{t_{1}^{r}}{\longrightarrow}\right)
$$

Combining (B.8)-(B.13), we can obtain the optimal timevarying road toll as follows: 


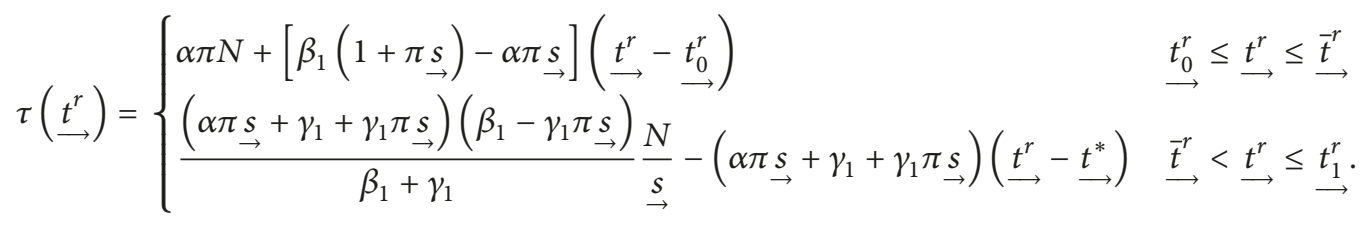

\section{User Equilibrium in Optimal Road Tolls with Duration Dependent Parking Fees}

In evening commuting of regime $o$, the individual travel cost of the commuter of early departing is

$$
C_{e}^{o}\left(t^{t^{o}}, \mu\right)=\beta_{2}\left(t^{t^{*}}-t^{t^{o}}\right)+\tau\left(t^{t^{o}}\right)+\mu\left(t^{t^{o}}-t^{\Delta}\right) .
$$

Here, $t^{o}$ is the departing time from workplace. The righthand side of (C.1) includes three terms; the first term is schedule delay cost, the second term is road toll, and the third term is duration dependent parking fee.

For the commuter of late departing, the individual travel cost is

$$
C_{e}^{o}\left(t^{o}, \mu\right)=\gamma_{2}\left(t^{o}-t^{*}\right)+\tau\left(t^{o}\right)+\mu\left(t^{o}-t^{\Delta}\right) .
$$

The equilibrium condition requires $\partial C_{e}^{o} / \partial t^{o}=0$, so the road toll rate can be obtained as follows:

$$
\frac{d \tau\left(\frac{t^{o}}{\longleftarrow}\right)}{d \stackrel{t^{o}}{\longleftarrow}}= \begin{cases}\beta_{2}-\mu & t_{0}^{o} \leq t^{o} \leq t^{*} \\ -\left(\gamma_{2}+\mu\right) & \stackrel{t^{*}}{\longleftarrow}<t^{o} \leq t_{1}^{o} .\end{cases}
$$

Using the method in Appendix B, we can also acquire the optimal starting time and ending time of rush hour:

$$
\begin{aligned}
& t_{0}^{o}=t^{*}-\frac{\gamma_{2}}{\beta_{2}+\gamma_{2}} \frac{N}{s}, \\
& t_{1}^{o}=t^{*}+\frac{\beta_{2}}{\beta_{2}+\gamma_{2}} \frac{N}{\leftarrow} .
\end{aligned}
$$

To keep a lowest no-negative toll level, the last commuter pays no toll, $\tau\left(t_{1}^{o}\right)=0$. In user equilibrium, the travel costs of the first and last commuter are equivalent:

$$
C_{e}^{o}\left(t_{0}^{o}, \mu\right)=C_{e}^{o}\left(t_{1}^{o}, \mu\right) .
$$

Given (C.1)-(C.5), the optimal time-varying road toll is obtained as

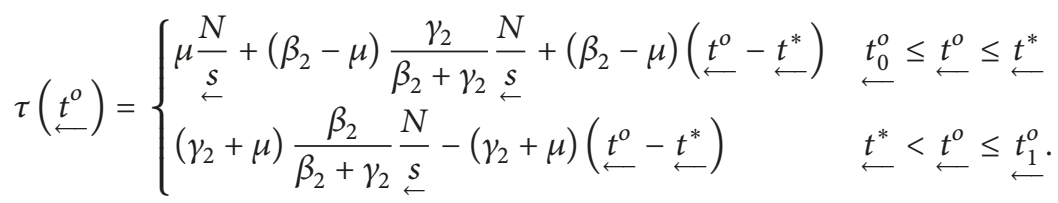

For morning commuting of regime $o$, the individual travel cost of the commuter who arrive early is

$$
\begin{aligned}
& C_{m}^{o}\left(\stackrel{t^{o}}{\longrightarrow}, \mu\right)=\alpha \pi \underset{s}{\stackrel{s}{\rightarrow}}\left(\stackrel{t^{o}}{\longrightarrow}-\underline{t_{0}^{o}}\right) \\
& +\beta_{1}\left(\stackrel{t^{*}}{\longrightarrow}-\stackrel{t^{o}}{\longrightarrow}-\pi \underset{s}{\rightarrow}\left(\stackrel{t^{o}}{\longrightarrow}-\underset{t_{0}^{o}}{\longrightarrow}\right)\right) \\
& +\tau\left(\stackrel{t^{o}}{\longrightarrow}\right) \\
& +\mu\left[t^{\Delta}-\stackrel{t^{o}}{\longrightarrow}-\pi \underset{s}{\rightarrow}\left(\stackrel{t^{o}}{\longrightarrow}-\stackrel{t_{0}^{o}}{\longrightarrow}\right)\right] .
\end{aligned}
$$

Here, $t^{o}$ is the departing time from bottleneck which is also the arrival time to parking lot. On the right-hand side of (C.7), the first term is the cost of searching time for parking spots, the second term is schedule delay cost, the third term is the road toll, and the fourth term is duration dependent parking fee.

For commuter who arrives late, the individual travel cost is

$$
\begin{aligned}
& C_{m}^{o}\left(\stackrel{t^{o}}{\longrightarrow}, \mu\right)=\alpha \pi \underset{\rightarrow}{s}\left(\stackrel{t^{o}}{\longrightarrow}-\underline{t_{0}^{o}}\right) \\
& +\gamma_{1}\left(\stackrel{t^{o}}{\longrightarrow}+\pi \stackrel{s}{\rightarrow}\left(\stackrel{t^{o}}{\longrightarrow}-\stackrel{t_{0}^{o}}{\longrightarrow}\right)-\underline{t^{*}}\right) \\
& +\tau\left(\stackrel{t^{o}}{\longrightarrow}\right) \\
& +\mu\left[t^{\Delta}-\stackrel{t^{o}}{\longrightarrow}-\pi \stackrel{s}{\rightarrow}\left(\stackrel{t^{o}}{\longrightarrow}-\underset{t_{0}^{o}}{\longrightarrow}\right)\right] .
\end{aligned}
$$


According to the equilibrium condition $d C_{m}^{o} / d \underline{t^{o}}=0$, we can get

$$
\begin{aligned}
& \frac{d \tau\left(\underline{t^{o}}\right)}{d \stackrel{t^{o}}{\longrightarrow}} \\
& = \begin{cases}\left(\beta_{1}+\mu\right)(1+\pi \underline{s})-\alpha \pi \underline{s} & \underline{t_{0}^{o}} \leq \underline{t^{o}} \leq \underline{\bar{t}^{o}} \\
-\left(\gamma_{1}-\mu\right)(1+\pi \underline{s})-\alpha \pi \stackrel{s}{\longrightarrow} & \stackrel{\overrightarrow{t^{o}}}{\longrightarrow} \leq \underline{t^{o}} \leq \underline{t_{1}^{o}} .\end{cases}
\end{aligned}
$$

The optimal beginning time and ending time of rush hour and the arrival time to parking lot of the commuter who arrives to the workplace on time are

$$
\stackrel{t_{0}^{o}}{\longrightarrow} \stackrel{t^{*}}{\longrightarrow}-\frac{\gamma_{1}(1+\pi \underset{s}{s})}{\beta_{1}+\gamma_{1}} \underset{\stackrel{N}{\rightarrow}}{\frac{N}{\rightarrow}},
$$

$$
\begin{aligned}
& \stackrel{t_{1}^{o}}{\longrightarrow}=t^{*}+\frac{\beta_{1}-\gamma_{1} \pi \stackrel{s}{\rightarrow}}{\beta_{1}+\gamma_{1}} \frac{N}{\stackrel{s}{\rightarrow}}, \\
& \stackrel{\vec{t}^{o}}{\rightarrow}=\stackrel{t^{*}}{\rightarrow}-\frac{\gamma_{1} \pi \stackrel{s}{\rightarrow}}{\beta_{1}+\gamma_{1}} \frac{N}{\stackrel{s}{\rightarrow}} .
\end{aligned}
$$

To keep a lowest no-negative toll level, the last commuter pays no toll, $\tau\left(t_{1}^{o}\right)=0$. In the user equilibrium, we also have

$$
C_{m}^{o}\left(\stackrel{t^{o}}{\longrightarrow}, \mu\right)=C_{m}^{o}\left(\stackrel{t^{o}}{\longrightarrow}, \mu\right)
$$

Given (C.7)-(C.11), we can obtain the optimal time-varying road toll presented as follows:

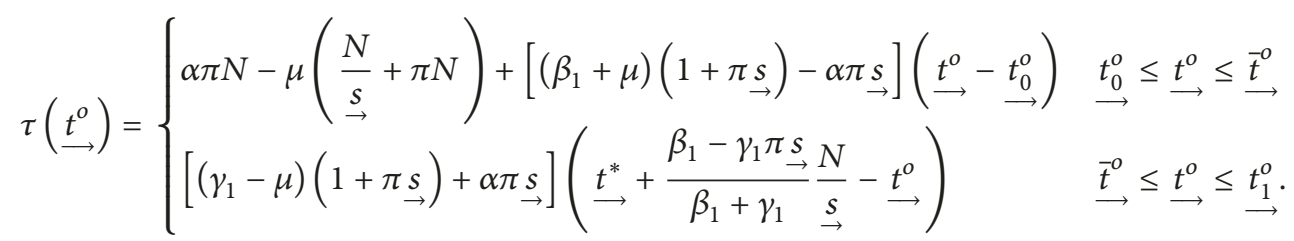

\section{Process of Comparing Parameter $L^{k}$}

According to the individual travel cost and system cost in morning and evening commutes of four regimes, we can obtain the parameters $K^{k}$ and $L^{k}$ which are given as follows:

$$
\begin{aligned}
& K^{f}=\frac{\beta_{2} \gamma_{2}}{\left(\beta_{2}+\gamma_{2}\right) \underset{\leftarrow}{s}}+\frac{\beta_{1} \gamma_{1}+\beta_{1}\left(\alpha+\gamma_{1}\right) \pi \underset{s}{\rightarrow}}{\left(\beta_{1}+\gamma_{1}\right) \underset{s}{s}}, \\
& K^{u} \\
& =\frac{\beta_{2} \gamma_{2}+\left(\beta_{2}-\gamma_{2}\right) \mu-\mu^{2}}{\left(\beta_{2}+\gamma_{2}\right) \stackrel{s}{\leftarrow}} \\
& +\frac{\left(\beta_{1}+\mu\right)\left(\gamma_{1}-\mu\right)}{\beta_{1}+\gamma_{1}} \frac{(1+\pi \stackrel{s}{\rightarrow})}{\stackrel{s}{\rightarrow}}+\frac{\alpha \pi\left(\beta_{1}+\mu\right)}{\beta_{1}+\gamma_{1}}, \\
& K^{r}=\frac{\beta_{2} \gamma_{2}}{\left(\beta_{2}+\gamma_{2}\right) \underset{\leftarrow}{s}}+\frac{\beta_{1} \gamma_{1}(1+\pi \underset{s}{s})}{\left(\beta_{1}+\gamma_{1}\right) \stackrel{s}{\rightarrow}}+\alpha \pi, \\
& K^{o} \\
& =\frac{\beta_{2} \gamma_{2}}{\left(\beta_{2}+\gamma_{2}\right) \stackrel{s}{\leftarrow}}+\frac{\beta_{2} \mu}{\left(\beta_{2}+\gamma_{2}\right) \stackrel{s}{\leftarrow}}+\frac{\beta_{1} \gamma_{1}(1+\pi \underset{s}{s})}{\left(\beta_{1}+\gamma_{1}\right) \stackrel{s}{\rightarrow}} \\
& +\alpha \pi-\frac{\mu \beta_{1}(1+\pi \underset{\rightarrow}{\rightarrow})}{\left(\beta_{1}+\gamma_{1}\right) \stackrel{s}{\rightarrow}},
\end{aligned}
$$

$$
\begin{aligned}
& L^{f}=\frac{\beta_{2} \gamma_{2}}{\left(\beta_{2}+\gamma_{2}\right) \underset{\leftarrow}{s}}+\frac{\beta_{1} \gamma_{1}+\beta_{1} \pi \underset{\rightarrow}{s}\left(\alpha+\gamma_{1}\right)}{\left(\beta_{1}+\gamma_{1}\right) \underset{s}{s}}, \\
& L_{e}^{u}(\mu) \\
& =\frac{\left[2 \alpha \beta_{2} \gamma_{2}+\beta_{2} \gamma_{2} \mu+\left(\beta_{2}-\gamma_{2}\right)\left(\mu^{2}+\alpha \mu\right)-\mu^{3}\right]}{2 \alpha\left(\beta_{2}+\gamma_{2}\right) \underset{\leftarrow}{s}}, \\
& L_{m}^{u}(\mu) \\
& =\frac{\beta_{1}\left(\gamma_{1}-\mu\right)}{\beta_{1}+\gamma_{1}} \frac{(1+\pi \underset{s}{\rightarrow})}{\stackrel{s}{\rightarrow}}+\frac{\alpha \beta_{1} \pi}{\beta_{1}+\gamma_{1}} \\
& +\frac{\mu(1+\pi s)}{2 \underset{\rightarrow}{s}}, \\
& L^{u}(\mu)=L_{e}^{u}(\mu)+L_{m}^{u}(\mu), \\
& L^{r}=\frac{1}{2} \frac{\beta_{2} \gamma_{2}}{\left(\beta_{2}+\gamma_{2}\right) \underset{\leftarrow}{s}}+\frac{1}{2} \alpha \pi+\frac{1}{2} \frac{\beta_{1} \gamma_{1}}{\beta_{1}+\gamma_{1}} \frac{(1+\pi \underset{s}{\rightarrow})}{\stackrel{s}{\rightarrow}}, \\
& L^{o}=\frac{1}{2} \frac{\beta_{2} \gamma_{2}}{\left(\beta_{2}+\gamma_{2}\right) \underset{\leftarrow}{s}}+\frac{1}{2} \alpha \pi+\frac{1}{2} \frac{\beta_{1} \gamma_{1}}{\beta_{1}+\gamma_{1}} \frac{(1+\pi \stackrel{s}{\rightarrow})}{\stackrel{s}{\rightarrow}} .
\end{aligned}
$$

Obviously, we can find that $L^{r}=L^{o}$. Here, the proof of $L^{f}>$ $L^{r}$ is given as follows: 


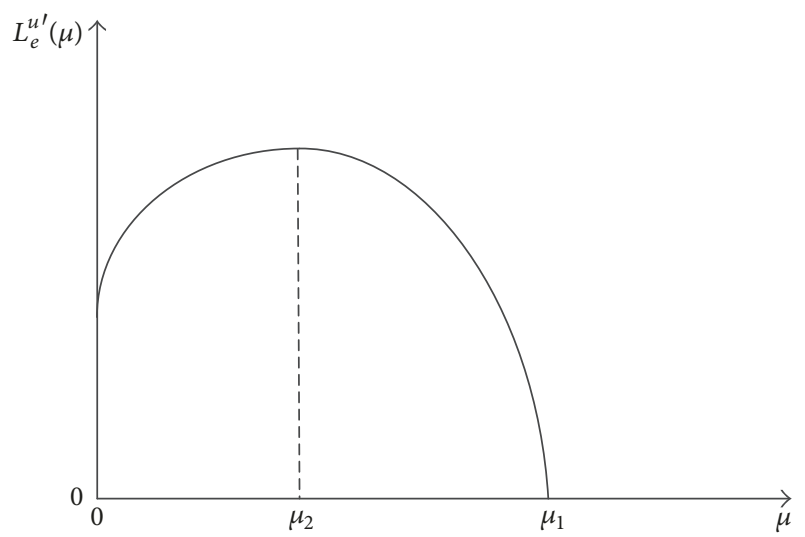

FIgURE 12: The first derivative function of parameter $L_{e}^{u}(\mu)$ in evening commuting.

$$
\begin{aligned}
L^{f}= & \frac{\beta_{2} \gamma_{2}}{\left(\beta_{2}+\gamma_{2}\right) \underset{\leftarrow}{s}}+\frac{1}{2} \frac{\beta_{1} \gamma_{1}(1+\pi \underset{\rightarrow}{s})}{\left(\beta_{1}+\gamma_{1}\right) \underset{s}{\rightarrow}} \\
& +\frac{1}{2} \frac{\beta_{1} \gamma_{1}(1+\pi \underset{s}{\rightarrow})}{\left(\beta_{1}+\gamma_{1}\right) \underset{s}{s}}+\frac{\beta_{1}}{\left(\beta_{1}+\gamma_{1}\right)} \alpha \pi .
\end{aligned}
$$

We have assumed $\beta_{1}(1+\pi s)>\alpha \pi s$ in Section 2.2 , so we have

$$
\begin{aligned}
L^{f}> & \frac{\beta_{2} \gamma_{2}}{\left(\beta_{2}+\gamma_{2}\right) \underset{\leftarrow}{\leftarrow}}+\frac{1}{2} \frac{\beta_{1} \gamma_{1}(1+\pi \underset{\rightarrow}{s})}{\left(\beta_{1}+\gamma_{1}\right) \underset{s}{\rightarrow}} \\
& +\frac{1}{2} \frac{\gamma_{1}}{\left(\beta_{1}+\gamma_{1}\right)} \alpha \pi+\frac{\beta_{1}}{\left(\beta_{1}+\gamma_{1}\right)} \alpha \pi \\
= & \frac{1}{2} \frac{\beta_{2} \gamma_{2}}{\left(\beta_{2}+\gamma_{2}\right) \underset{\leftarrow}{s}}+\frac{1}{2} \frac{\beta_{1} \gamma_{1}(1+\pi \underset{s}{\rightarrow})}{\left(\beta_{1}+\gamma_{1}\right) \underset{s}{\rightarrow}}+\frac{1}{2} \alpha \pi \\
& +\frac{1}{2} \frac{\beta_{2} \gamma_{2}}{\left(\beta_{2}+\gamma_{2}\right) \underset{\leftarrow}{s}}+\frac{1}{2} \frac{\beta_{1}}{\left(\beta_{1}+\gamma_{1}\right)} \alpha \pi \\
= & L^{r}+\frac{1}{2} \frac{\beta_{2} \gamma_{2}}{\left(\beta_{2}+\gamma_{2}\right) \underset{s}{s}}+\frac{1}{2} \frac{\beta_{1}}{\left(\beta_{1}+\gamma_{1}\right)} \alpha \pi .
\end{aligned}
$$

And in regime $u, L^{u}(\mu)$ is a strictly increasing function with respect to parking fee rate $\mu, 0 \leq \mu<\alpha-\beta_{1}$; here $\alpha-\beta_{1}=$ $\alpha-\gamma_{2}$; it means that $L^{u} \geq L^{f}$. This can be proved as follows.

Proof. By solving $L_{e}^{u \prime}\left(\mu_{1}\right)=0, L_{e}^{u \prime \prime}\left(\mu_{2}\right)=0$, we can obtain that $\mu_{1}=\left(\left(\beta_{2}-\gamma_{2}\right)+\sqrt{\left(\beta_{2}-\gamma_{2}\right)^{2}+3\left(\alpha \beta_{2}+\beta_{2} \gamma_{2}-\alpha \gamma_{2}\right)}\right) / 3$, $\mu_{2}=\left(\beta_{2}-\gamma_{2}\right) / 3$. We could easily get $\mu_{1}>\mu_{2}$.

Figure 12 shows the diagram of the first derivative of parameter $L_{e}^{u}$. For the first derivative of $L_{e}^{u}$, if $0 \leq \mu \leq$ $\mu_{1}, L_{e}^{u^{\prime}}(\mu)>0$ and if $\mu>\mu_{1}, L_{e}^{u^{\prime}}(\mu)<0$. For the second derivative of $L_{e}^{u}$, if $0 \leq \mu \leq \mu_{2}, L_{e}^{u \prime \prime}(\mu) \geq 0$ and if $\mu>$ $\mu_{2}, L_{e}^{u \prime \prime}(\mu)<0$.
Scenario 1. If $a-\gamma_{2} \leq \mu_{1}, L_{e}^{u^{\prime}}\left(\alpha-\gamma_{2}\right) \geq 0$. Then $L_{e}^{u}(\mu)$ is monotone increase when $0 \leq \mu<a-\gamma_{2}$. For the morning commuting of regime $u, L_{m}^{u}{ }^{\prime}=(1 / 2)\left(\left(\gamma_{1}-\beta_{1}\right) /\left(\beta_{1}+\gamma_{1}\right)\right)((1+$ $\pi \stackrel{s}{\rightarrow}) / \stackrel{s}{\rightarrow})$. We have that $L_{m}^{u}(\mu)$ increases as $\mu$ increases. So $L^{u}(\mu)$ is a monotone increasing function in regime $u$.

Scenario 2. If $a-\gamma_{2}>\mu_{1}$, then $L_{e}^{u^{\prime}}\left(\alpha-\gamma_{2}\right)<0$. We could solve the first derivative of $L^{u}(\mu)$ with respect to parking fee rate $\mu$ when $\mu=a-\gamma_{2}$ :

$$
\begin{aligned}
& L^{u^{\prime}}\left(\alpha-\gamma_{2}\right)=L_{e}^{u^{\prime}}\left(\alpha-\gamma_{2}\right)+L_{m}^{u \prime}\left(\alpha-\gamma_{2}\right), \\
& L^{u^{\prime}}\left(\alpha-\gamma_{2}\right)=\frac{1}{2} \frac{\left(-3 \alpha^{2}-\gamma_{2}^{2}+3 \alpha \beta_{2}-\beta_{2} \gamma_{2}+3 \alpha \gamma_{2}\right)}{\alpha\left(\beta_{2}+\gamma_{2}\right) \underset{\leftarrow}{s}} \\
& \quad+\frac{1}{2} \frac{\gamma_{1}-\beta_{1}}{\beta_{1}+\gamma_{1}} \frac{(1+\pi s)}{\stackrel{s}{\rightarrow}}, \\
& L^{u \prime}\left(\alpha-\gamma_{2}\right)=\frac{1}{2 \alpha\left(\beta_{2}+\gamma_{2}\right) \underset{\leftarrow}{\leftarrow}}\left[\left(3 \alpha \beta_{2}-3 \alpha^{2}\right)\right. \\
& \left.\quad+\left(\alpha \beta_{2}-\beta_{2} \gamma_{2}\right)+\left(2 \alpha \gamma_{2}-\gamma_{2}^{2}\right)+a\left(\beta_{2}-\gamma_{2}\right) \pi \underset{\leftarrow}{s}\right] .
\end{aligned}
$$

Obviously, each term on the right hand of (D.6) is positive and the first derivative of $L^{u}$ with respect to parking fee rate $\mu$ when $\mu=\alpha-\gamma_{2}$ is greater than zero, $L^{u^{\prime}}\left(\alpha-\gamma_{2}\right)>0$.

When $\mu_{2}<\mu<a-\gamma_{2}$, the second derivative of $L^{u}$ in evening commuting with respect to parking fee rate is less than zero, $L_{e}^{u^{\prime \prime}}(\mu)<0$; then we have $L_{e}^{u^{\prime}}(\mu)>L_{e}^{u^{\prime}}\left(\alpha-\gamma_{2}\right)$ and $L_{m}^{u}{ }^{\prime}(\mu)=L_{m}^{u}{ }^{\prime}\left(\alpha-\gamma_{2}\right)=(1 / 2)\left(\left(\gamma_{1}-\beta_{1}\right) /\left(\beta_{1}+\gamma_{1}\right)\right)((1+$ $\pi \stackrel{s}{\rightarrow}) / \underset{s}{\rightarrow})>0$; then we have $L^{u^{\prime}}(\mu)>L^{u^{\prime}}\left(\alpha-\gamma_{2}\right)>0$.

When $0 \leq \mu \leq \mu_{2}$, the first derivatives of $L_{e}^{u}(\mu)$ and $L_{m}^{u}(\mu)$ are larger than zero, $L_{e}^{u \prime}(\mu)>0$ and $L_{m}^{u}{ }^{\prime}(\mu)>0$, so the first derivatives of $L^{u}$ with respect to parking fee rate are greater than zero, $L^{u^{\prime}}(\mu)>0$, and $L^{u}(\mu)$ is a monotone increasing function in regime $u$.

\section{Notations}

$t^{*}$ : The desired departing time from workplace in the evening commuting

$t^{k}$ : The departing time from workplace in regime $k, k=\{f, u, r, o\}$

$t_{0}^{k}$ : The departing time from workplace of the first commuters in regime $k, k=\{f, u, r, o\}$

$t_{1}^{k}$ : The departing time from workplace of the last commuters in regime $k, k=\{f, u, r, o\}$

$C_{e}^{k}$ : The individual travel cost in the evening commuting in regime $k, k=\{f, u, r, o\}$

$\mathrm{SC}_{e}^{k}$ : The system cost in the evening commuting in regime $k, k=\{f, u, r, o\}$

$\tilde{N}^{k}$ : The number of commuters who depart early from workplace in regime $k, k=\{f, u, r, o\}$

$r_{1}^{k}$ : Departure rate from workplace of early departing in regime $k, k=\{f, u, r, o\}$ 
$r_{2}^{k}$ : Departure rate from workplace of late departing in regime $k, k=\{f, u, r, o\}$

$s$ : The capacity of bottleneck in work-to-home direction

$t^{*}$ : The desired arrival time to workplace in the morning commuting

$t^{k}$ : The leaving time from bottleneck in regime $k, k=\{f, u, r, o\}$

$t_{0}^{k}$ : The arrival time to parking lot of the first commuter

$\longrightarrow \quad$ in regime $k, k=\{f, u, r, o\}$

$\bar{t}^{k}$ : The arrival time to parking lot of the commuter who arrives to the workplace on time in regime $k, k=\{f, u, r, o\}$

$t_{1}^{k}$ : The arrival time to parking lot of the last commuter in regime $k, k=\{f, u, r, o\}$

$C_{m}^{k}$ : The individual travel cost in the morning commuting in regime $k, k=\{f, u, r, o\}$.

$\mathrm{SC}_{m}^{k}$ : The system travel cost in the morning commuting in regime $k, k=\{f, u, r, o\}$

$s$ : The capacity of bottleneck in home-to-work

$\rightarrow$ direction

$\sim k$

$\tilde{N}$ : The number of commuters who arrive early to workplace in regime $k, k=\{f, u, r, o\}$

$r_{1}^{k}$ : Departure rate from home of early arrival in regime $k, k=\{f, u, r, o\}$

$r_{2}^{k}$ : Departure rate from home of late arrival in regime $k, k=\{f, u, r, o\}$.

\section{Conflicts of Interest}

The authors declare that they have no conflicts of interest regarding the publication of this paper.

\section{Acknowledgments}

This study is substantially supported by National Key Project of Research and Development of China (Grant no. 2016YFC0802505), the National Natural Science Foundation Council of China through Project [no. 71531011], and a research grant of Shanghai Shuguang Program [Project no. 13SG23].

\section{References}

[1] H. Wang and X. Zhang, "Game theoretical transportation network design among multiple regions," Annals of Operations Research, vol. 249, no. 1-2, pp. 97-117, 2017.

[2] R. Arnott, A. de Palma, and R. Lindsey, "A temporal and spatial equilibrium analysis of commuter parking," Journal of Public Economics, vol. 45, no. 3, pp. 301-335, 1991.

[3] G. N. Bifulco, "A stochastic user equilibrium assignment model for the evaluation of parking policies," European Journal of Operational Research, vol. 71, no. 2, pp. 269-287, 1993.

[4] E. Verhoef, P. Nijkamp, and P. Rietveld, "The economics of regulatory parking policies: the (im)possibilities of parking policies in traffic regulation," Transportation Research Part A: Policy and Practice, vol. 29, no. 2, pp. 141-156, 1995.
[5] X. Zhang, H. Yang, and H.-J. Huang, "Improving travel efficiency by parking permits distribution and trading," Transportation Research Part B: Methodological, vol. 45, no. 7, pp. 1018-1034, 2011.

[6] Z. S. Qian, F. E. Xiao, and H. M. Zhang, "The economics of parking provision for the morning commute," Transportation Research Part A: Policy and Practice, vol. 45, no. 9, pp. 861-879, 2011.

[7] W. Vickrey, "Congestion theory and transport investment," American Economic Review, vol. 59, pp. 251-261, 1969.

[8] C. F. Daganzo, "The uniqueness of a time-dependent equilibrium distribution of arrivals at asingle bottleneck," Transportation Science, vol. 19, no. 1, pp. 29-37, 1985.

[9] R. Arnott, A. de Palma, and R. Lindsey, "A structural model of peak-period congestion: a traffic bottleneck with elastic demand," The American Economic Review, vol. 83, no. 1, pp. 161179,1993

[10] X. Zhang, H. M. Zhang, and L. Li, "Analysis of user equilibrium traffic patterns on bottlenecks with time-varying capacities and their applications," International Journal of Sustainable Transportation, vol. 4, no. 1, pp. 56-74, 2010.

[11] N. Geroliminis and C. F. Daganzo, "Existence of urban-scale macroscopic fundamental diagrams: some experimental findings," Transportation Research Part B: Methodological, vol. 42, no. 9, pp. 759-770, 2008.

[12] R. Arnott, "A bathtub model of downtown traffic congestion," Journal of Urban Economics, vol. 76, no. 1, pp. 110-121, 2013.

[13] M. Fosgerau, "Congestion in the bathtub," Economics of Transportation, vol. 4, no. 4, pp. 241-255, 2015.

[14] N. Geroliminis, "Cruising-for-parking in congested cities with an MFD representation," Economics of Transportation, vol. 4, no. 3, pp. 156-165, 2015.

[15] W. Liu and N. Geroliminis, "Modeling the morning commute for urban networks with cruising-for-parking: An MFD approach," Transportation Research Part B: Methodological, vol. 93, pp. 470-494, 2016.

[16] Y. Ji, M. Xu, H. Wang, and C. Tan, "Commute Equilibrium for Mixed Networks with Autonomous Vehicles and Traditional Vehicles," Journal of Advanced Transportation, vol. 2017, Article ID 6218363, 10 pages, 2017.

[17] N. Jiang, "Optimal signal design for mixed equilibrium networks with autonomous and regular vehicles," Journal of Advanced Transportation, vol. 2017, Article ID 5649823, 13 pages, 2017.

[18] A. de Palma and R. Lindsey, "Comparison of morning and evening commutes in the Vickrey bottleneck model," Transportation Research Record, no. 1807, pp. 26-33, 2002.

[19] C. F. Daganzo, "System optimum and pricing for the daylong commute with distributed demand, autos and transit," Transportation Research Part B: Methodological, vol. 55, pp. 98117, 2013.

[20] E. J. Gonzales and C. F. Daganzo, "The evening commute with cars and transit: Duality results and user equilibrium for the combined morning and evening peaks," Transportation Research Part B: Methodological, vol. 57, pp. 286-299, 2013.

[21] H. Yang, W. Liu, X. Wang, and X. Zhang, "On the morning commute problem with bottleneck congestion and parking space constraints," Transportation Research Part B: Methodological, vol. 58, pp. 106-118, 2013.

[22] J. Wang, X. Zhang, and H. M. Zhang, "Parking permits management and optimal parking supply considering traffic 
emission cost," Transportation Research Part D: Transport and Environment, 2016.

[23] X. Zhang, H.-J. Huang, and H. M. Zhang, "Integrated daily commuting patterns and optimal road tolls and parking fees in a linear city," Transportation Research Part B: Methodological, vol. 42, no. 1, pp. 38-56, 2008.

[24] X. Zhang and B. van Wee, "Efficiency comparison of various parking charge schemes considering daily travel cost in a linear city," European Journal of Transport and Infrastructure Research, vol. 11, no. 2, pp. 234-255, 2011.

[25] H. Wang, X. Wang, and X. Zhang, "Dynamic resource allocation for intermodal freight transportation with network effects: Approximations and algorithms," Transportation Research Part B: Methodological, vol. 99, pp. 83-112, 2017.

[26] H. Wang, W. H. K. Lam, X. Zhang, and H. Shao, "Sustainable Transportation Network Design with Stochastic Demands and Chance Constraints," International Journal of Sustainable Transportation, vol. 9, no. 2, pp. 126-144, 2015.

[27] K. A. Small, "The scheduling of consumer activities: work trips," The American Economic Review, vol. 72, no. 3, pp. 467-479, 1982.

[28] R. Arnott and J. Rowse, "Modeling parking," Journal of Urban Economics, vol. 45, no. 1, pp. 97-124, 1999. 


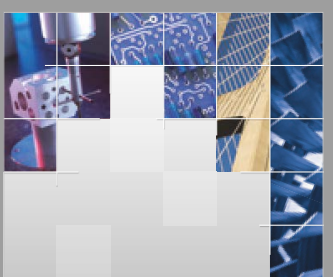

\section{Enfincering}
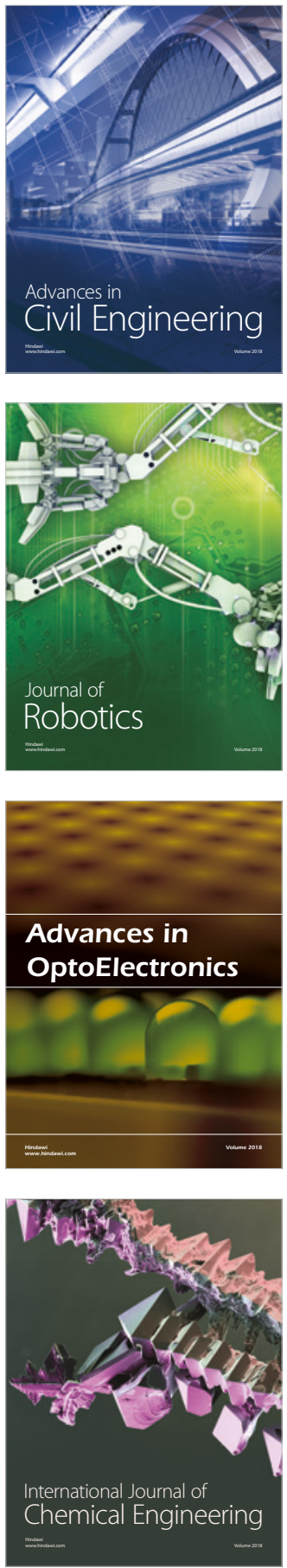

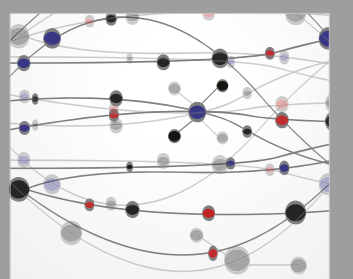

\section{Rotating \\ Machinery}

The Scientific World Journal

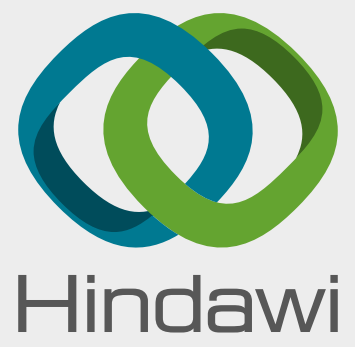

Submit your manuscripts at

www.hindawi.com
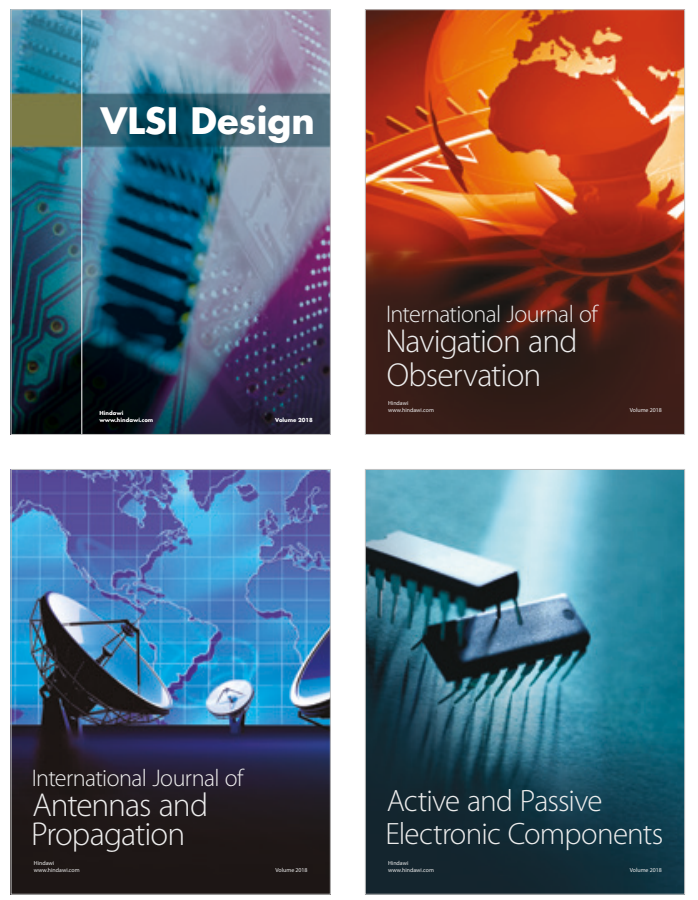
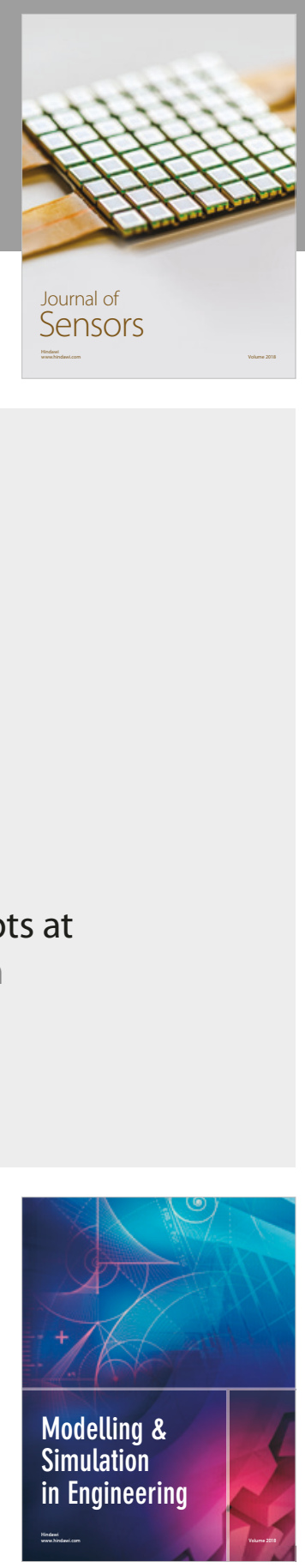

\section{Advances \\ Multimedia}
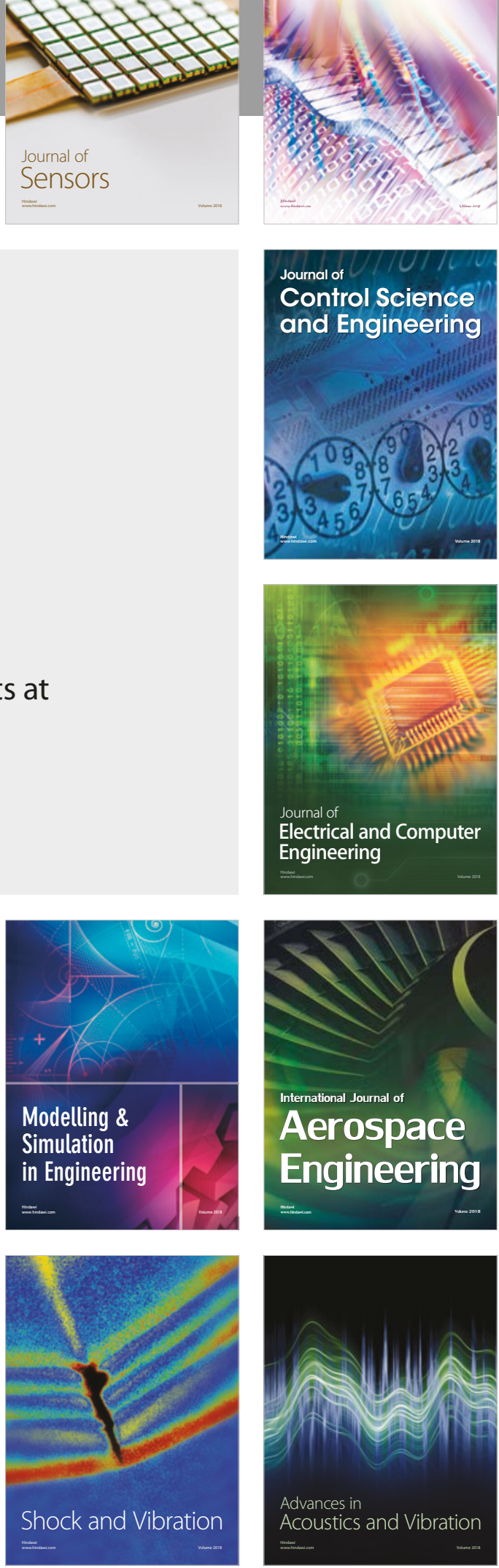